POS PROCEEDINGS

\title{
Recent Standard Model results in ATLAS and CMS
}

\section{Pierre-Hugues Beauchemin**}

Tufts University

E-mail: hugo.beaucheminetufts.edu

A summary of ATLAS and CMS QCD and electroweak related Standard Model measurements is presented with emphasis on recent results.

Corfu Summer Institute 2017 'School and Workshops on Elementary Particle Physics and Gravity' 2-28 September 2017

Corfu, Greece

* Speaker.

†n behalf of the ATLAS and CMS Collaborations 


\section{Introduction}

The challenge of our generation in High Energy Physics (HEP) is to find New Physics, i.e. evidence that there is a theory Beyond the Standard Model (BSM) that is needed to make sense of experimental data. So far it didn't happen. The Standard Model (SM) of particle physics has been successful at explaining all results obtained at collider experiments (and others) so far. The entire particle spectrum included in the SM has fully been directly and unambiguously observed, the last missing piece being the Higgs boson discovered at the LHC in 2012 [1, 2]. The structure of the three fundamental interactions included in the SM has long been confirmed to a high level of precision. The most striking example certainly comes from quantum electrodynamics where predictions of the electron magnetic moment at a five-loop accuracy have been experimentally verified [3]. Precision measurements of electroweak parameters such as the mass of the Z-boson $\left(\Delta M_{Z} \sim 0.002 \%\right)$, the width of the Z-boson $\left(\Delta \Gamma_{Z} \sim 0.09 \%\right)$ or the weak mixing angle $\left(\Delta \sin ^{2} \theta_{W} \sim 0.5 \%\right)$ provide a few of many more examples [4] that established the Standard Model as the best theory we have in HEP.

Over the last a few decades, a large part of the physics program of various collider experiments consisted of looking for significant deviations between experimental measurement results and SM predictions, with the expectations of isolating phenomena, which could only be explained by one of the BSM theories in the market (or yet to be formulated). The synergetic efforts between the theory and the experimental HEP communities aiming at the development and the observation of physics beyond the SM does however not account for the bulk of HEP research effort. An important part of this work, both in the theoretical and the experimental communities, consists of sophisticated and precise predictions or measurements of SM processes. The question is then: why, if the SM is so successful and has been confirmed to a high level of accuracy, there is still the need to further develop and explore this theory?

One obvious answer is that the SM predicts many rare processes that have not yet been observed, but which would be background to some of the New Physics processes predicted by interesting BSM extensions. For example, vector boson scattering processes predicted by the SM have not yet been experimentally measured but could reveal the existence of extra quartic gauge couplings not included in the SM. Accurately predicting and measuring these SM rare processes is therefore one of the reasons why work is dedicated in HEP to further explore and develop the SM. In general, measuring precisely some of the SM parameters, especially those characterizing the weak interaction, and looking for deviations with respect to the SM predictions, is a powerful way for discovering new phenomena, justifying work toward the SM in HEP. However, the most serious efforts in SM-related research activities, both from the theory and the experimental perspectives, comes from studies of the strong interaction. In the following, we will briefly explain why QCD measurements are critical for HEP. We will then expand a little more on the relevance of electroweak interaction measurements at the Large Hadron Collider (LHC), before discussing some of the most important and interesting experimental results obtained by the ATLAS and CMS Collaborations on QCD and electroweak physics.

\subsection{The Strong Interaction at Hadron Colliders}

The strong interaction intervenes in various ways and at various scales in every single event at hadron colliders. Of course, the matrix element (Feynman diagrams) of the processes of interest 
might include a strong interaction vertex. QCD would therefore be required for predicting cross sections for these processes. However, even if no such vertex is present in the Feynman diagrams of interest, the fact that the initial state particles of any collision at hadron colliders involve quarks and gluons, implies that the strong interaction plays a role in the description of any hadron collider event. Predictions indeed need to account for parton distribution functions (PDF), soft parton emission from initial or final state quarks and gluons, parton fragmentation and hadronization in jets of particles, and spectator hadronic activity, referred to as underlying events ${ }^{1}$. While the processes described by the matrix-element calculations happen at highly virtual energy scales $\left(Q^{2}\right)$, where the strong coupling constant $\alpha_{S}$ is large, and so where perturbative calculations can be made, parton distribution functions, fragmentation, hadronization, and underlying event phenomena happen at a much lower $Q^{2}$ scale, where perturbative calculations are not possible because of the too high value taken by the strong coupling constant $\alpha_{S}$. This is however not a hindrance to the predictability of LHC phenomena thanks to the factorization theorem. It states that the probabilities for large distance and short distance processes factorize. The large distance factors are universal and can be obtained from ancillary measurements, before being convoluted to the small-distance matrixelement calculations. This allows for testable predictions of any processes occurring at hadron colliders.

Additional complications come from the fact that these large-distance phenomena don't fully scale, i.e. that they need to be "evolved" from a $Q^{2}$ scale characterizing large distances, to a higher energy scale describing short distance physics. This can be done, for the PDFs, by a differential equation, the DGLAP equation, while the partonic system can be "evolved" using a parton shower algorithm. Once again, QCD has the elements to address these predictability issues. There are however problems that lie in the details of these solutions that save the predictive power of QCD.

The main issue comes from the fact that the theories used to describe the various QCD effects discussed above involve assumptions, approximations, simplifications, and phenomenological models (not obtained from first principles) that impact the final state selections and differential cross section predictions. For example, parton showers are formulated in the soft and collinear approximation, where QCD radiation is enhanced, therefore underestimating hard jet production. In addition, parton showers can only approximatively cure the effect of the first two powers of large logarithms that undermine perturbative calculations obtained from matrix elements (approximate Next-to-Leading-Log resummation). Finally, the description of the final states predicted using a parton shower depends on the choice of parameters used in the algorithm. For example, the kinematic of the jets obtained from a parton shower algorithm would vary with the choice of the ordering parameter that determines the kinematic hierarchy of the parton emitted in their evolution from small to large scales (and vice versa). PDFs are also a significant source of uncertainty on any LHC predictions. As mentioned above, they must be extracted from ancillary measurements before being convoluted to the predictions obtained from matrix-element calculations. These ancillary measurements involve both statistical and systematic uncertainties that are then propagated to the main LHC predictions. There are also theoretical assumptions that are used to extract the PDFs from ancillary data and to evolve them to the scale at which matrix-element calculations have been made. These constitute further sources of uncertainties on any LHC predictions. Finally,

\footnotetext{
${ }^{1}$ A more complete description of these effects is, for example, provided in $[5,6,7]$.
} 
to obtain a complete description of hadronic events, phenomenological models involving ad hoc assumptions and relying on many free parameters are used to predict the impact of fragmentation, hadronization, and underlying events on the predictions. All these assumptions, simplifications, and approximations result in uncertainties assigned to any LHC predictions and measurements. These uncertainties can often be larger than a new physics signal to be observed over SM expectations. Such uncertainties on QCD prediction could therefore mask a new physics discovery at hadron colliders, generate a false signal, suppress the sensitivity of data analysis to new physics, or simply impede the interpretation of a new physics signal, would a discovery be made. This is the main problem with QCD.

These predicaments can however be mitigated by a better theoretical description of the various QCD effects, which require more precise and sophisticated experimental measurements of QCD for testing the theoretical improvements, and for exploring aspects that require new developments. It is therefore necessary, for the possibility of a successful discovery program at the LHC, to devote efforts at better understanding and improving QCD.

\subsection{The Weak Interaction at Hadron Colliders}

The SM contains 26 parameters: 12 fermion masses, 3 coupling constants, 9 matrix elements and phases, and 2 Higgs parameters. There are however only 17 of these that need to be measured because of the dependence predicted by the SM between many of these parameters. Measuring all the parameters therefore allow to exploit the redundancy to reveal potential inconsistencies between measured values, possibly hinting for new physics. In that context, new precise measurements of electroweak parameters give more stringent consistency tests, hopefully leading to a discovery. Even if the scale at which new physics would occur is much larger than the weak scale directly accessible at the LHC, electroweak relationships and parameters can be used to define observables in which new physics can be probed through virtual loop effects. For example, the measurement of a non-zero value for the three oblique parameters $\mathrm{S}, \mathrm{T}$, and $\mathrm{U}$, parametrizing gauge boson selfenergies, would provide a clear evidence for BSM physics [8]. In order to discover new physics from such virtual effects, very precise SM measurements are needed.

It is certainly possible for physics beyond the SM to be described by a theory not yet formulated. This possibility does however not hinder the search strategy of the LHC. For example, the framework of effective field theory can be used to parametrize new physics effect at low energy in a generic way, by adding higher dimensional operators to the SM Lagrangian. A plethora of new physics operators can be tested in this somehow model-independent way. This is the idea behind the precision measurement of triple and quartic gauge couplings: deviation with respect to the SM predictions would reveal the effect of such higher dimensional operators.

From these two categories of exemple, we can see that, for different reasons than those used to justify QCD measurements, precise experimental and theoretical investigations of the SM electroweak sector are necessary for the success of the LHC discovery mission.

\subsection{Adopted strategies}

The focus below will be on the knowledge gained on the SM from the experimental measurements in ATLAS and in CMS. In order to enhance our knowledge of the SM from these measurements, the general adopted strategy is essentially the same for all the examples reported below. This 
strategy consists of choosing to measure a set of differential cross section observables for which different SM theoretical predictions disagree with each other, and to use measurement results to determine which calculation provides the best description of the data, and which theoretical improvements are needed. This is illustrated in Figure 1, where we can see that the Pythia 8 [9], the Herwig++ [10], and the MadGraph+Pythia8 [11] predictions for the azimuthal angular decorrelation $\left(\Delta \phi_{12} \neq \pi\right)$ between the two leading jets in inclusive 2-jets events are in complete disagreement. The results of the measurement of this quantity obtained by the CMS Collaboration [12], however, demonstrates that the MadGraph+Pythia8 prediction is much better than the two others at describing the data. It can therefore be concluded from this measurement that parton showers provide a poor description of azimuthal angular decorrelation data, especially at large angles, but that matching a parton shower to a matrix element successfully yields a good description of such phenomena. This is not unexpected in view of the progresses that have been made over the last 15 years of QCD studies, but it illustrates well the strategy put in place by experimental collaborations in order to provide crucial information about the strong interaction from their observations.

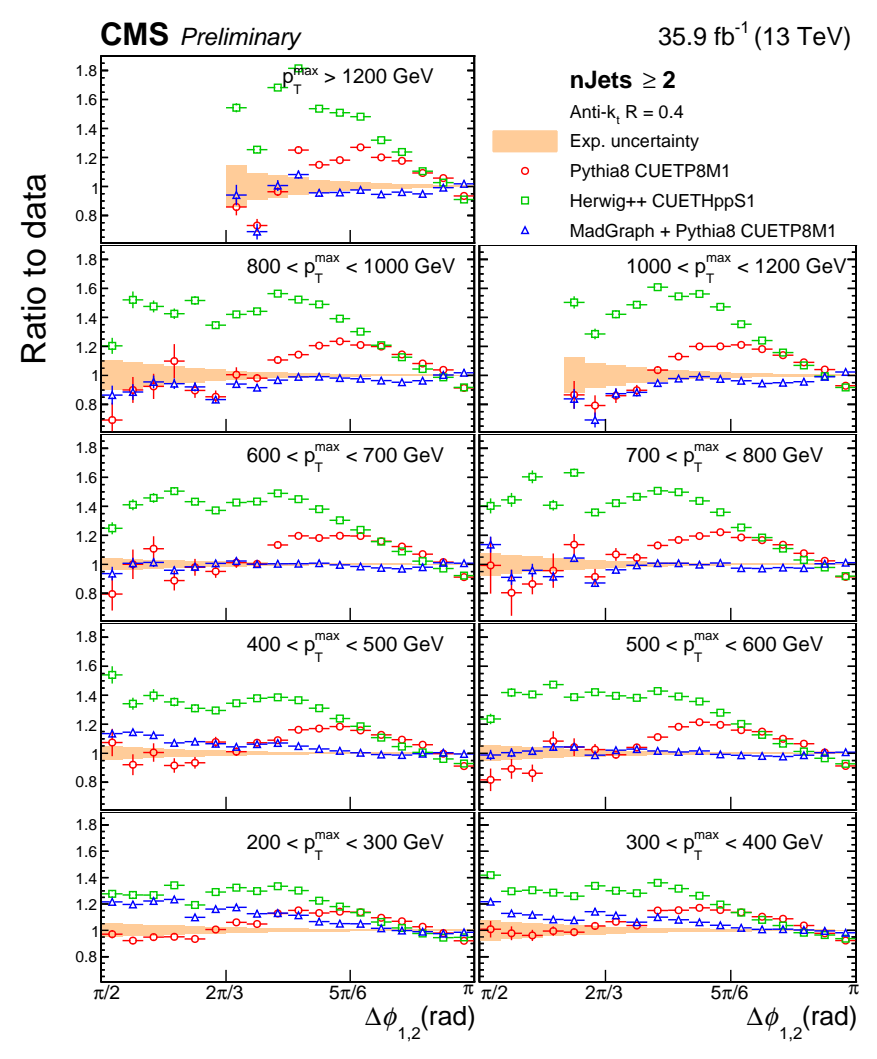

Figure 1: The azimuthal angular decorrelation $\left(\Delta \phi_{12} \neq \pi\right)$ between the two leading jets in inclusive 2-jets events as measured by the CMS collaboration [12].

In the following, a sample of QCD measurement results, illustrating some of the most interesting recent LHC observations, will be presented. Note however that with over 120 results for each experiment, it is impossible to cover all the SM measurements that have been done by ATLAS 
and CMS in Run 1 and Run 2. It is also impossible to discuss in detail the measurements to be presented below. The focus will therefore be on some of the most striking or puzzling results that have been published by ATLAS and CMS in 2016-2018. Results are gathered according to QCD or electroweak physics most relevant to each measurement. In the next section, results about PDF studies will be presented, followed by those related to the understanding of soft parton radiation (QCD Bremsstrahlung). Section 4 will then focus on phenomena involving hard parton emission. The last two sections of this proceeding will elaborate on the measurements of processes dominated by the electroweak interaction and on the measurements of electroweak parameters respectively.

\section{Parton Distribution Functions}

$\mathrm{W}$ and $\mathrm{Z}$ total and differential cross section measurements are used in PDF fits to improve the description and the precision of light-quark (u, d, s) PDFs. These measurements are also highly valuable for purely experimental reasons in that they provide reference cross sections for luminosity and charged lepton transverse momentum $\left(p_{T}\right)$ calibration, and provide an empirically meaningful validation of the experimental tools used in many other measurements. The dominant source of uncertainty $(1.8 \%)$ affecting the ATLAS absolute cross section results [13, 14] comes from independent luminosity estimates used to derive the measured cross section values. Taking the ratio of the total inclusive cross sections obtained with $\mathrm{W}^{+}$and $\mathrm{W}^{-}$events allows for a cancelation of such uncertainty, leading to a total precision of $0.4 \%$. This is sufficient for in-depth tests of lightquark PDF modeling. As can be seen in Figure 2, ATLAS data feature an excellent agreement with the JR14 and NNPDF3.0 using Run 1 data. However, refinements of these PDFs led Run 2 ATLAS data to favor CT14nnlo and MMHT14nnlo68CL PDFs. It is interesting to contrast this to the conclusions that can be drawn from differential cross section measurement results. For example, ATLAS $7 \mathrm{TeV}$ results [13] for the measurement of the $\mathrm{W}^{-}$charged lepton pseudo-rapidity (Figure 3 left) and CMS $8 \mathrm{TeV}$ results [16] for the W charge asymmetry (Figure 3 right) both favor the ABM12 PDF set over the others. This is not necessarily a contradiction. These PDF sets have many common features. The objective of all these measurements is not so much to find the best PDF but to use these measurement results in the various PDF fits, improving the agreement between each PDF central value and the data and reducing the size of the PDF uncertainty band. This is illustrated in Figure 4 where light-quark valance PDFs including the LHC data in the fit are compared to the PDFs before the LHC data inclusion for various $x$ values. We can see in this figure that the LHC data modify the central value of the light-quark PDFs, especially at low $x$, and also reduces the overall PDF uncertainty. Each of the PDF sets that roughly agree with the data can be improved this way.

The biggest impact ATLAS W and Z inclusive cross section measurements had on PDFs however comes from the description of strange-quark PDFs. The inclusion of the 2010 ATLAS W and Z cross section measurement results in s-quark PDF fits indicated an enhancement of the strangeness contribution to PDFs compared to what was obtained in neutrino-induced charged-current deep inelastic scattering experiments [15]. Before the ATLAS results, these PDFs were poorly known at low- $x$ due to the restricted kinematics in fixed-target experiments. Nuclear effects also made the PDF extraction from fixed-target experiments more complicated and less precise. ATLAS results were therefore acceptable, but they needed confirmation. Repeating the measurement with 

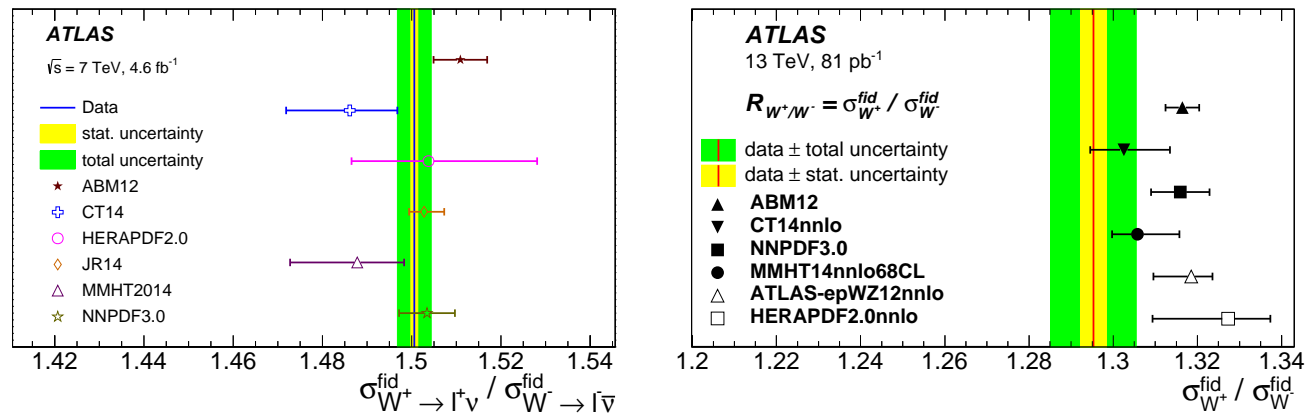

Figure 2: Comparison of the inclusive $W^{+}$to $W^{-}$fiducial cross section ratio between theoretical predictions using various PDF models to the ATLAS (left) $7 \mathrm{TeV}$ data, and (right) $13 \mathrm{TeV}$ data [14].
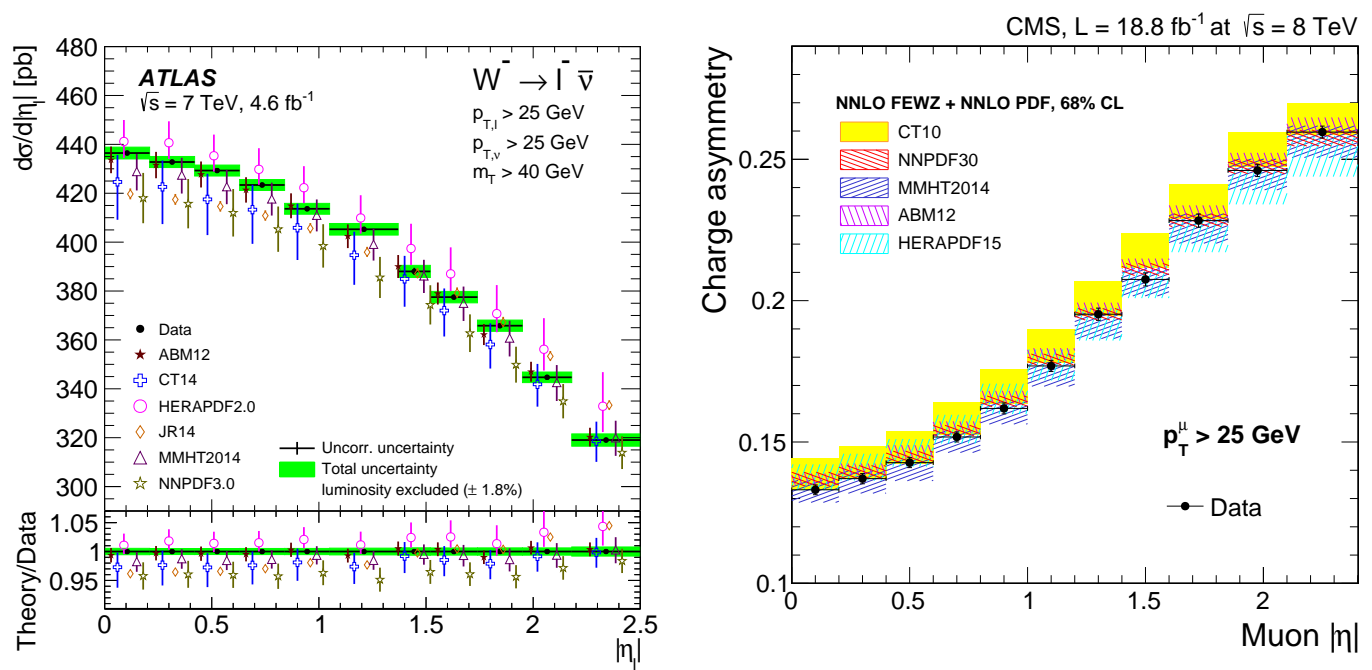

Figure 3: Left: ATLAS measurement [13] of the $W^{-}$differential cross section as a function of the charged lepton pseudorapitiy and comparison with different PDF predictions. Right: CMS measurement [16] of the $W$ charge asymmetry as a function of the muon pseudorapitiy and comparison with different PDF predictions.

the full 2011 dataset, the ATLAS Collaboration dramatically improved the knowledge of s-quark PDFs and confirmed the strangeness enhancement observed in 2010, suggesting a restoration of the SU(3) flavor symmetry in the sea distribution. These results are respectively presented in the left and right panels of Figure 5.

The result of inclusive jet cross section measurements can also be used to improve our knowledge of PDFs, especially of the gluon PDFs. As can be seen in Figure 6 (left), jet double differential inclusive $p_{T}$ vs. rapidity distributions obtained from perturbative QCD (pQCD) predictions describe all the generic features of the LHC data for the corresponding distribution up to the $\mathrm{TeV}$ scale, covering many orders of magnitude in cross sections [17]. The measurement results are so precise that NNLO calculations are needed to describe the data, as can be seen on the right panel of Figure 6 [18]. These data can therefore be included in PDF fits in order to improve our 

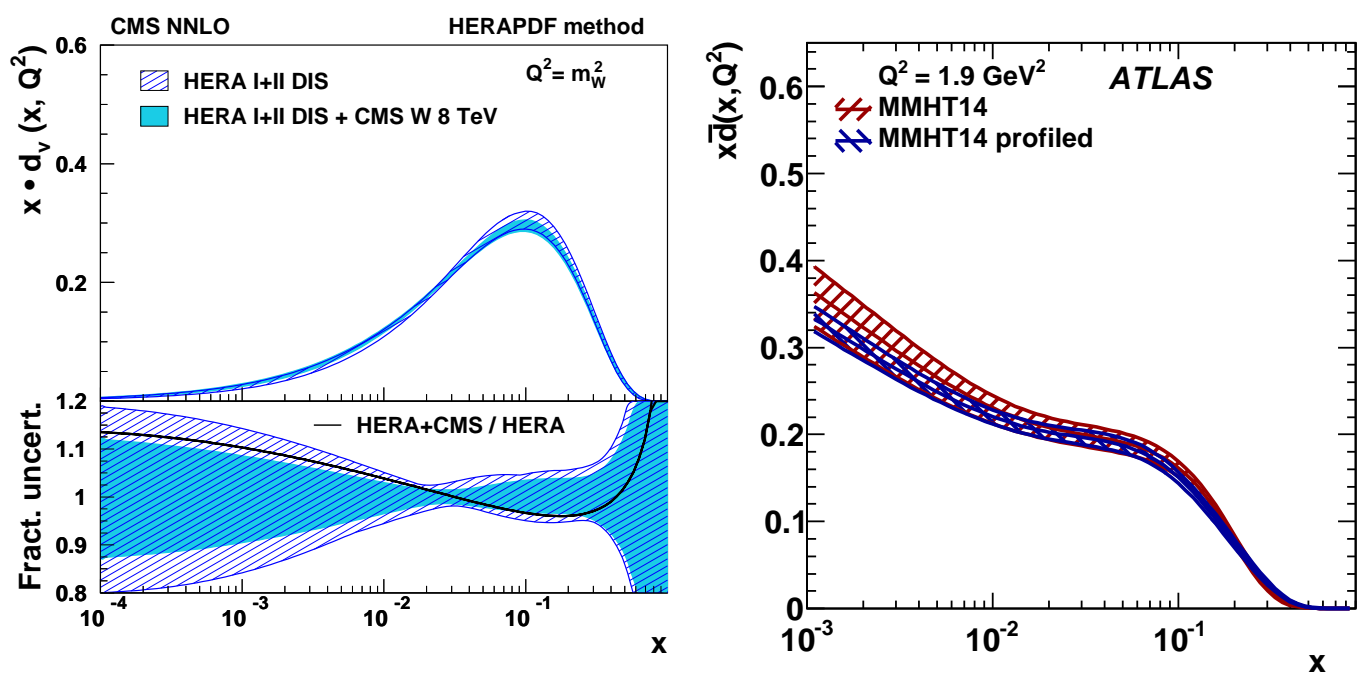

Figure 4: Left: Impact of inclusive $\mathrm{W}$ cross section results with CMS $8 \mathrm{TeV}$ data on the $d$-quark HERAPDF set [16]. Right: Impact of the W/Z inclusive cross section results with ATLAS $7 \mathrm{TeV}$ data on $\bar{d}$-quark MMHT14 PDF set [13].
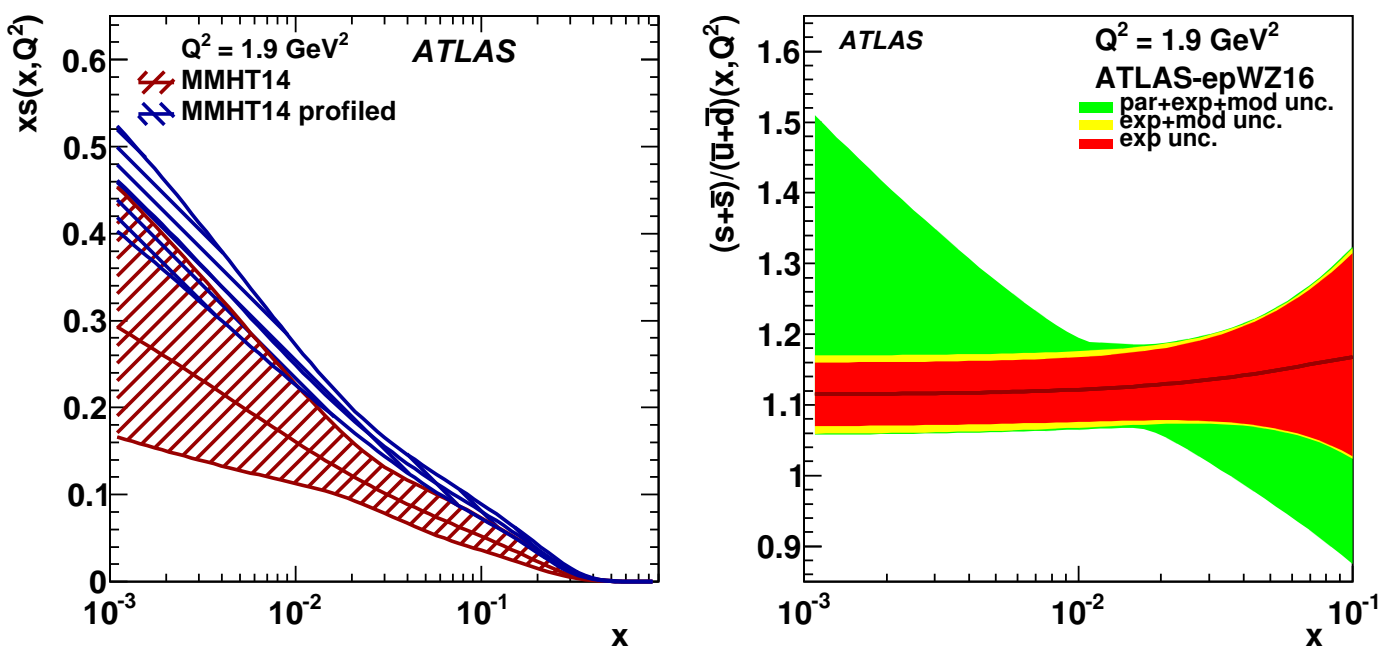

Figure 5: Left: Distribution of the strange-quark $x_{S}$ PDFs as a function of Bjorken- $x$ at a scale of $Q_{0}^{2}=1.9$ $\mathrm{GeV}^{2}$ for the MMHT14 PDF set before and after profiling (PDF and its uncertainty at the min of the $\chi^{2}$ quantifying the compatibility of the data with the theory). Right: Ratio $R_{s}(x)$ of the strange-quark to the light-sea-quark contribution to the PDFs from the present ATLAS-epWZ16 determination at the starting scale $Q_{0}^{2}=1.9 \mathrm{GeV}^{2}$. Both results are obtained from [13]. 
knowledge of the gluon PDFs. This is particularly striking at high- $x$, where triple differential cross section measurements performed by both the ATLAS and the CMS Collaborations led to an order of magnitude reduction in the gluon PDF systematic uncertainty for processes at both low and high $Q^{2}$. This is illustrated in Figure 7 with the CMS results [17]. To obtain such level of precision, the correlations between all systematic uncertainties in the experimental measurements needed to be accounted for. All these results demonstrate how important ATLAS and CMS data are for the knowledge of the structure of protons.
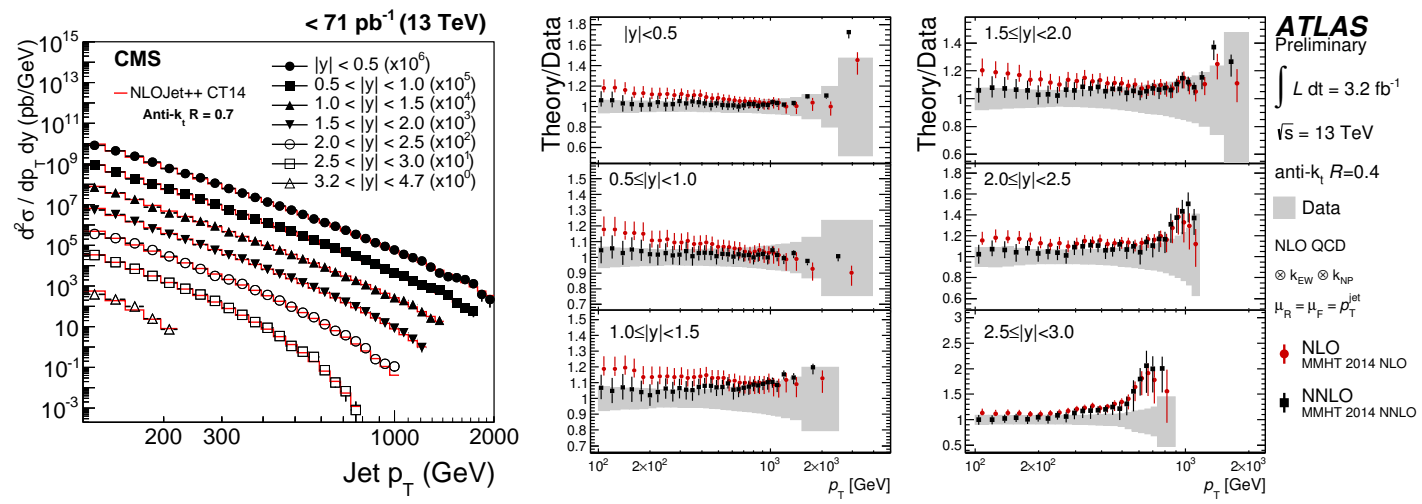

Figure 6: Left: Comparison of CMS double-differential inclusive jet cross section measurement as function of jet $p_{T}$ with NLOJet++ predictions for different jet rapidity ranges [17]. Right: Ratios of the NLO and NNLO pQCD predictions to the measured inclusive jet cross-sections on ATLAS 13 data [18], shown as a function of the jet $p_{T}$ in six lyl bins for anti-kt jets with $\mathrm{R}=0.4$.
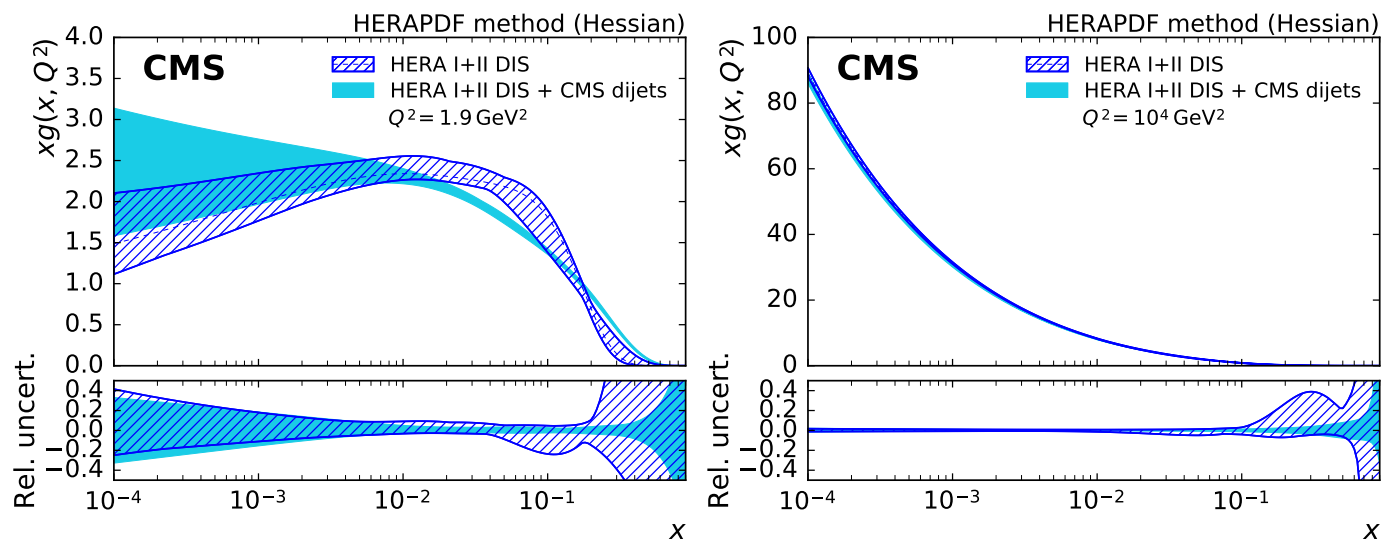

Figure 7: The gluon PDFs as a function of $\mathrm{x}$ as derived from HERA inclusive DIS data alone (hatched band) and in combination with CMS dijet data (solid band) [17]. The PDFs are shown with their total uncertainties at the evolution scales $Q^{2}=1.9 \mathrm{GeV}^{2}$ and $Q^{2}=10^{4} \mathrm{GeV}^{2}$ (right).

\section{Soft Parton Radiation}

Precise measurements of the $\mathrm{W}$-boson or the Z-boson transverse momentum distributions provide tests of multiple aspects of QCD phenomena. The position of the peak of this distribution in- 
forms about the intrinsic $k_{T}$ of the partons within the protons, one of the parameters used to model large-distance physics included in each theoretical predictions at hadron colliders. In addition, the tail of the transverse momentum distribution can be used to test higher order pQCD corrections to $\mathrm{W}$ and $\mathrm{Z}$ matrix-element calculations, probing the (N)NLO level of accuracy. However, the main importance of such measurement is to use the low to moderate $p_{T}$ kinematic regime to test the large logarithms resummation at up to Next-to-Next-to-Leading-Log (NNLL) accuracy, or equivalently, to test and tune initial state radiation parton shower models used in all other hadron collider predictions. The $p_{T}^{Z}$ ATLAS measurements made with the large $20 \mathrm{fb}^{-1} 8 \mathrm{TeV}$ dataset [20] lead towards many important conclusions. As can be seen on the left panel of Figure 8, NNLL soft-gluon resummation performed with RESBOS ${ }^{2}$ provides a good description of the data at low and average $p_{T}^{Z}$, but not when hard gluon emission dominates. In this high- $p_{T}$ regime, however, fixed-order predictions are not better at explaining the data, even when the NNLO accuracy is reached, as can be seen on the right panel of Figure 8. Similar conclusions are obtained from pure parton shower modeling of QCD radiation. It is therefore crucial to use a fixed-order matrix-element calculation matched to a parton shower, even if the matrix element is only available at the leading order, in order to be able to describe vector boson transverse momenta at larger values. Note that the predictions widely vary with the choice of a parton shower model and its free parameter values (see left of Figure 9). The $p_{T}^{Z}$ observable is therefore highly valuable for testing parton shower improvements and tuning its parameters. Once a a good description of soft QCD emission is established with one process, it can be used in the prediction of a wide range of other processes. For example, as demonstrated by the CMS Collaboration with their measurement of the ratio of the Z-boson $p_{T}$ to the W-boson $p_{T}$ [22], a shower validated with $\mathrm{Z}$ events could be used to describe $\mathrm{W}$ events with high accuracy, even if the matrix element features some differences between the two processes at higher order. This is illustrated on the right panel of Figure 9.

Tuning is, in a sense, a way to mitigate modeling defects by leaving sufficient freedom to the parametrization used in the model for it to provide an accurate description of the data. This is illustrated in Figure 10. On the right panel of this figure, the Pythia parton shower has been tuned to the ATLAS Z-boson $p_{T}$ measurement presented above. The predictions, before and after tuning, are compared to the measurement result of a different quantity than the tuned one: the $\phi^{\star}$ observable, correlated but more precise than $p_{T}^{Z}$ [24]. The agreement after tuning is excellent. The left panel of Figure 10 is just the opposite procedure: the Pythia parton shower has been tuned with a measurement of $\phi^{\star}$, and the quality of the tune is tested against the $p_{T}^{Z}$ measurement. Once again, tuning allows for an excellent description of the data. It is important to realize that on the right panel, the matrix-element calculation is provided by Pythia, so it is a leading order calculation. On the left panel, the Pythia parton shower is merged to the NLO Powheg matrixelement calculation [25]. Even if Powheg+Pythia provides a better description of the data before tuning, there is no difference between the two predictions after tuning. Tuning therefore takes away defects on QCD modeling, no matter what they are.

The importance and impact of tuning must however not be overstated. First of all, $p_{T}^{Z}$ and $\phi^{\star}$

\footnotetext{
${ }^{2}$ RESBOS is an approximate NNLO prediction because it uses NNLO Wilson coefficient functions to apply corrections to initial state radiation calculations. RESBOS also uses the GNW parametrization at low $p_{T}$ to model nonperturbative effects. It does however not include $\gamma^{*}$ contribution, leading to significant disagreement with the data for $p_{T} \sim 50 \mathrm{GeV}$ when the mass of the Z-boson is in the range $46 \mathrm{GeV}<M_{l l}<66 \mathrm{GeV}$. [21]
} 

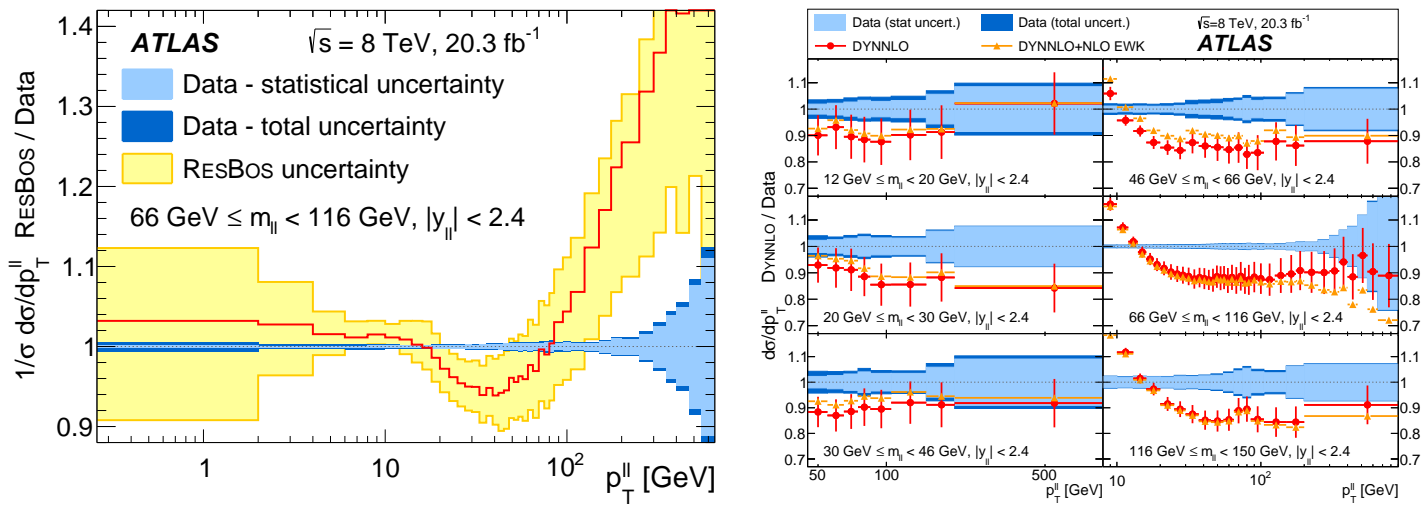

Figure 8: Ratio of ResBos (left) or DYNNLO (right) theoretical predictions to the combined Born-level ATLAS $8 \mathrm{TeV}$ measurement results for the Z-boson normalized $p_{T}$-differential cross $\operatorname{section}\left(\frac{1}{\sigma}\right) \frac{d \sigma}{d p_{T}(\ell \ell)}[20]$. For the comparison to the ResBos predictions, the leptons are required to have an invariant mass consistent with the Z-boson mass peak ( $66 \mathrm{GeV}<M_{\ell \ell}<116 \mathrm{GeV}$ ). DYNNLO [23] predictions are compared with the data in six different $M_{\ell \ell}$ regions. In all cases, the rapidity of the leptons is required to satisfy $\left|y_{\ell \ell}\right|<2.4$.
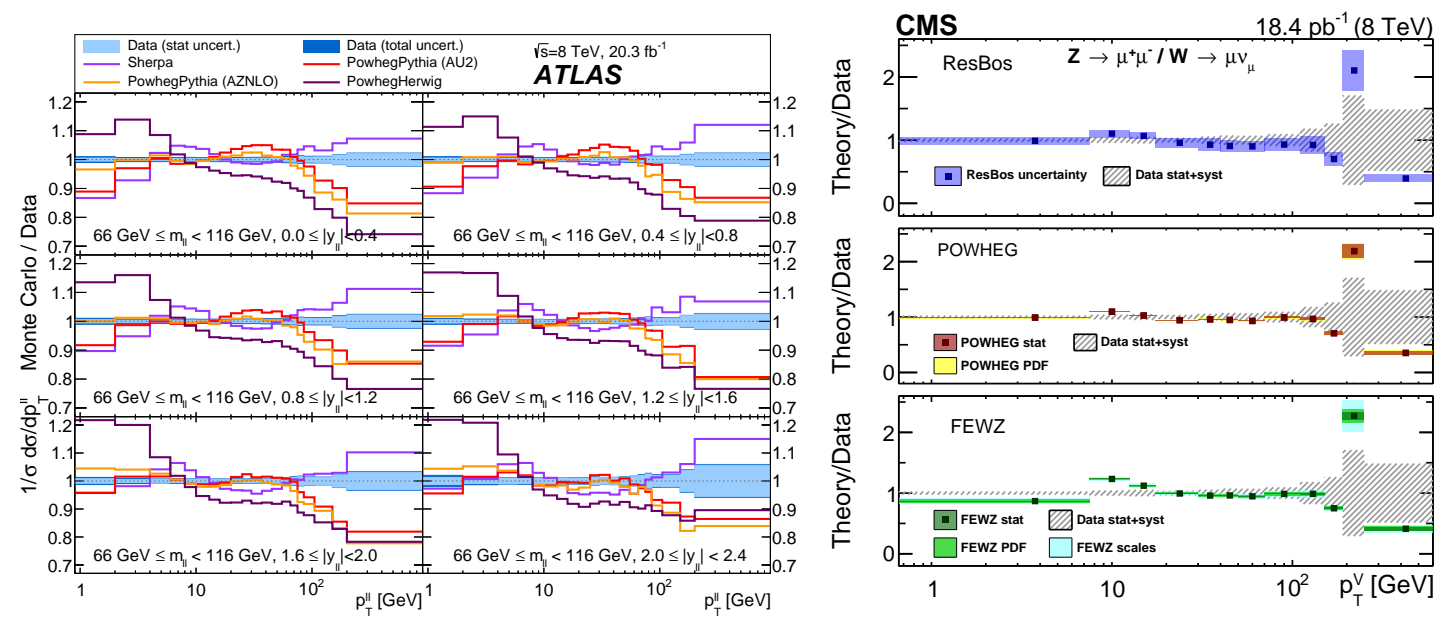

Figure 9: Left: The ratio of $\left(\frac{1}{\sigma}\right) \frac{d \sigma}{d p_{T}(\ell \ell)}$ as predicted by various NLO MC generators (matched to a parton shower) to the combined Born-level ATLAS $8 \mathrm{TeV}$ data, in different $\left|y_{\ell \ell}\right|$ ranges for events at the Z-boson mass peak [20]. Right: CMS measurement of the normalized $p_{T}$ differential cross section ratio of $\mathrm{Z}$ to $\mathrm{W}$ for muon channel compared with theoretical predictions [22].

are highly correlated. The agreement with data is in general imperfect when tuning and testing are made on very different observables: tuning provides a better description of the data featuring a similar event topology than the one used in the tuning process. This is illustrated in Figure 11. The AZNLO tune presented above has been obtained from the $7 \mathrm{TeV}$ ATLAS Z $p_{T}$ and $\phi^{\star}$ measurements selecting Z-boson candidates with an invariant mass in the range $66 \mathrm{GeV}<m_{l l}<116 \mathrm{GeV}$. The middle panel of Figure 11 shows that testing this tune against the independent $8 \mathrm{TeV}$ ATLAS data yields an excellent agreement: the tune describes well this kind of event topology. However, 

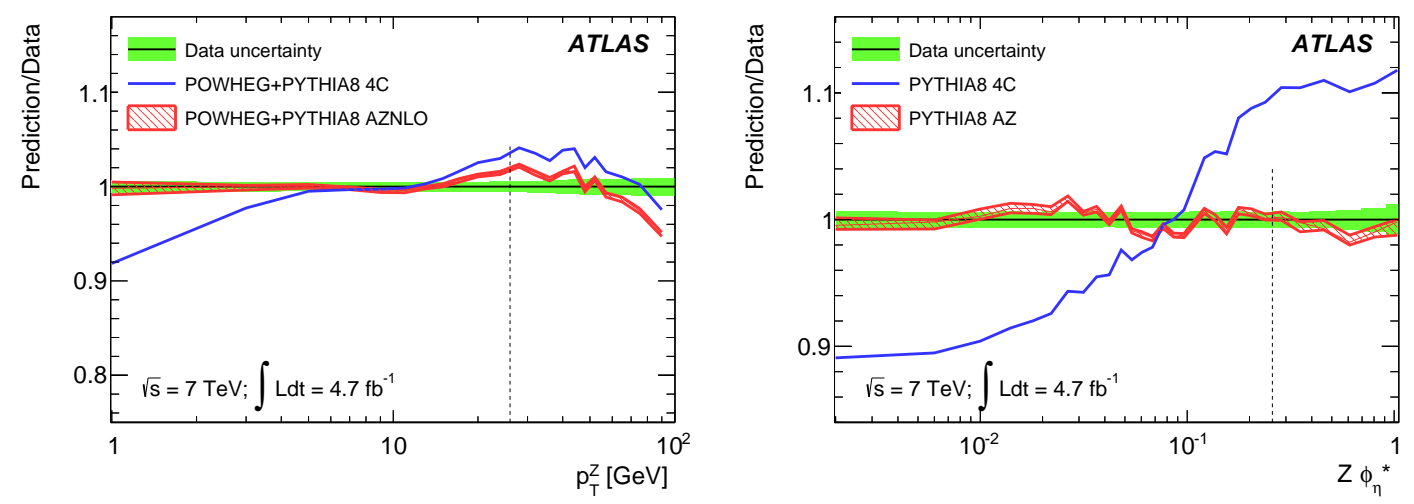

Figure 10: Left: Comparison of the Powheg+Pythia8 $p_{T}^{Z}$ differential cross section predictions for the default Pythia8 AC tune to the AZNLO tune, obtained from ATLAS $7 \mathrm{TeV} \phi^{\star}$ data [24]. Right: Comparison of the Pythia8 $\phi^{\star}$ differential cross section predictions for its default AC tune to the AZNLO tune, obtained from ATLAS $7 \mathrm{TeV} p_{T}^{Z}$ data.

when looking at the same $\phi^{\star}$ distribution, but for Z-boson candidates with an invariant mass in the range $46 \mathrm{GeV}<m_{l l}<66 \mathrm{GeV}$ (upper panel), the old tune provides a better description of the data than the new tune. The value of a tune is therefore of very limited scope, and it cannot be the solution to all QCD emission mis-modeling issues for future predictions. Improving the models provides a more general, robust, and stable solution than hiding the defects with a dedicated tune.

Other observables than $p_{T}^{Z}$ and $\phi^{\star}$ are more directly related to the soft QCD radiation. This is the case of $k_{T}$-splitting scales. Parton radiation is intimately related to jet clustering algorithms: each step of the $k_{T}$ algorithm [26] identifies the pair of partons which would most likely proceed from QCD emission or splitting. Sequential $k_{T}$-type jet algorithms therefore produce infrared- and collinear-safe branching histories of partons. This is, for example, used in the CKKW-L merging procedure to determine when a branching occurs. Measuring the $k_{T}$-splitting scales at different steps of the corresponding jet clustering iteration would allow testing resummation, parton shower, merging procedure, and higher order matrix-element corrections at different scales. We can define a hierarchy of such $k_{T}$-splitting scales as the scale for which the number of inputs to a jet clustering drops from $k+1$ to $k$ particles. This scale is noted as $\sqrt{d_{k}}$. As such, large $k$ and low $\sqrt{d_{k}}$ values are sensitive to soft emission and to non-perturbative effects; low $k$ and high $\sqrt{d_{k}}$ are sensitive to hard radiation.

Figure 12 presents the results of the ATLAS $k_{T}$-splitting scale measurements on $\mathrm{W} \rightarrow \ell v$ events [27]. The left panel features a measurement of $\sqrt{d_{0}}$. Interesting conclusions can be obtained from it. The results confirm that low $\sqrt{d_{0}}$ region is sensitive to the choice of a parton shower model, and it is therefore ideal for testing potential improvements in soft QCD radiation modelings. The high $\sqrt{d_{0}}$ region is not sensitive to the modeling details of soft QCD effects, but it demonstrates that the data are better described by high-multiplicity predictions, matched to a parton shower, even if the matrix elements are only calculated at tree-level, than by the low-multiplicity predictions obtained at an NLO accuracy. Combining a high-multiplicity matrix-element calculation at NLO with a parton shower would therefore be the best way to generally improve the predictions. This is 


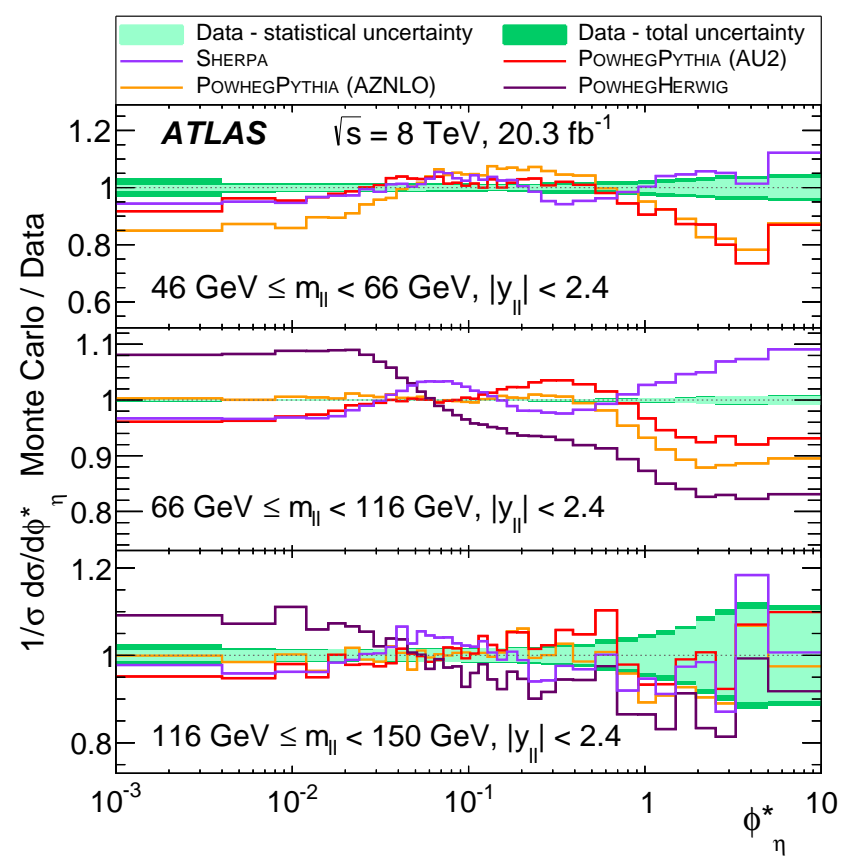

Figure 11: The ratio of $\left(\frac{1}{\sigma}\right) \frac{d \sigma}{d \phi_{\eta}^{\star}}$ as predicted by various MC generators to the combined Born-level ATLAS $8 \mathrm{TeV}$ data, in the three different regions of $m_{\ell \ell}$ for $\left|y_{\ell \ell}\right|<2.4[20]$.

what is attempted by the Sherpa community with their MEPS@NLO prescription [28]. The right panel presents the measurement results of the ratio $\sqrt{\frac{d_{1}}{d_{0}}}$, which is more precise than any of the $k_{T^{-}}$ splitting scale measurements. Such a ratio is sensitive to parton showers and NLO matrix-element matching procedures. As can be seen in Figure 12, the MC@NLO matching strategy provides a better description of these data than the Powheg approach.

An alternate approach to parton shower (or analytical resummation) developments for describing events with important soft QCD radiation activity is to strip the soft radiation off the event, leaving only the hard activity. This is what the soft drop grooming procedure achieves. Soft drop is a jet grooming procedure that eliminates the non-global logs due to the correlation between the inand out-of-jet scales, which prevents parton shower from going beyond the LL accuracy [30]. By applying the soft drop algorithm to jets, the soft portions of a jet are removed, resulting in a dramatically reduced Sudakov peak in the jet mass distribution, showing that very soft and collinear radiation has been removed. The soft drop procedure therefore yields NNLL resummed jet observables. The CMS Collaboration applied this technique to a jet mass measurement [29]. As can be seen in Figure 13, after the soft drop grooming procedure is applied, there is almost no difference between different parton shower models and between parton showers and explicit NNLL resummation predictions for a wide range of jet mass values. The comparison with the data also reveals that the Sudakov correction has been suppressed as expected. This grooming procedure is therefore interesting for studying hard processes without bias due to soft QCD effects. 

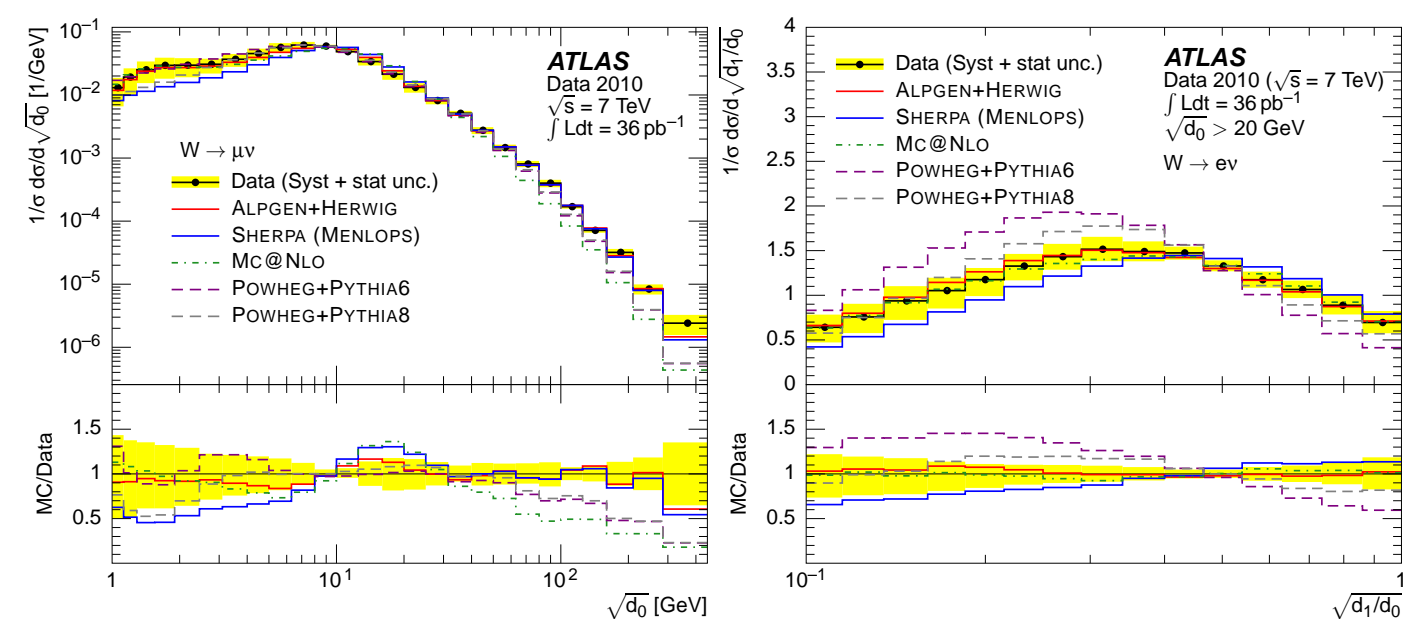

Figure 12: Comparison of the $7 \mathrm{TeV}$ ATLAS measurements of the $k_{T}$-splitting scale $\left.\sqrt{(} d_{0}\right)$ distribution in the Wmunu channel (left) and of the ratio of the $k_{T}$-splitting scales $\sqrt{d_{1} / d_{0}}$ in the Wenu channel (right) to the predictions from various MC generators [27].

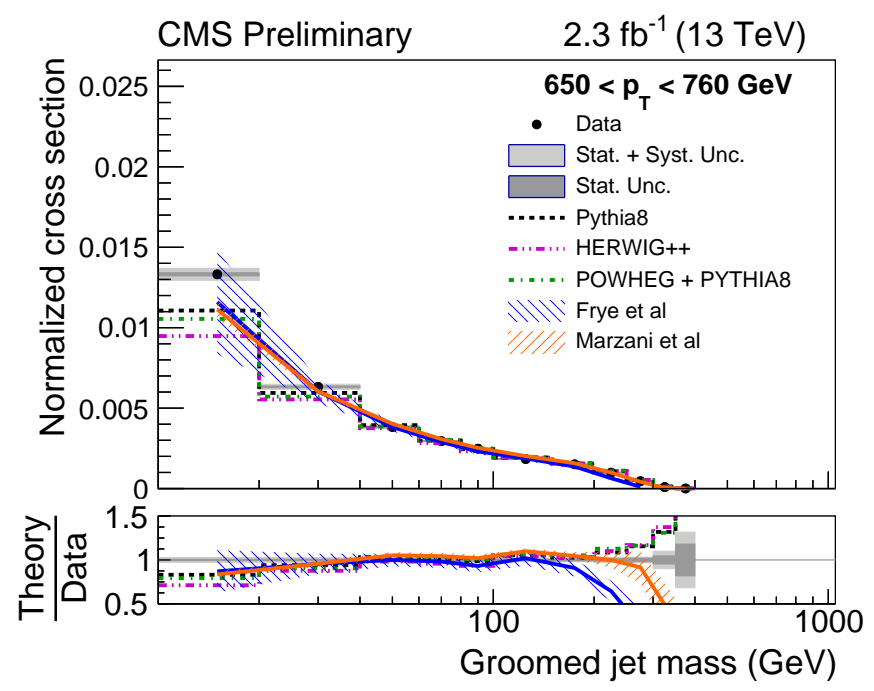

Figure 13: Results of the unfolded groomed jets mass using $13 \mathrm{TeV}$ CMS data [29] with jets of $650 \mathrm{GeV}<$ $p_{T}<760 \mathrm{GeV}$ and comparisons to Pythia8, Herwigg++ and Powheg+Pythia predictions as well as with two analytic NNLL resummation calculations obtained by Frye et al. [30] and Marzani et al. [31] respectively.

\section{Hard Parton Emissions}

Searches for new physics frequently require very hard jets in the events. The accuracy of the modeling of such hard QCD activity can be studied in $\mathrm{W}$ or $\mathrm{Z}$ events with more exclusive final states. At the LHC, there is a very large phase space available for QCD Bremsstrahlung, leaving room for $\mathrm{pQCD}$ hard radiation to be studied with large datasets. To study these effects, one simply needs to select events for which the vector boson is accompanied by a certain number of high 
momentum jets. The predictions for such events involve different challenges than what has been considered above for inclusive observables. When jets are explicitly required in the final state, both the initial and the final state radiation gets entangled. However, the biggest challenge comes from the fact that requiring jets in a final state results in the realization of multiple very different energy scales in each event, forcing more sophisticated predictions.

The number of observables that can provide important information about pQCD in this context is huge. The simplest one is probably the jet multiplicity itself. ATLAS [32] and CMS [33] jet multiplicity results, obtained from $13 \mathrm{TeV}$ W/Z+jets measurements, are presented in Figure 14. These results provide important information on how best to model the hard QCD radiation. For example, parton showers lack of large angle hard emission. A good description of the data therefore requires matching between a matrix-element calculation, describing the hard jets in the event, and a parton shower, to describe the soft jet activity. Experimental results indicate that a better description of the data is obtained from matrix-element calculations involving a large number of jets, even if calculated only at leading order, than a fewer hard jets calculated at NLO. Similar conclusions have been obtained with the $k_{T}$-splitting scale analyses. Finally, the high jet multiplicity region provides an interesting opportunity to test possible improvements in the merging strategy used to complement matrix-element predictions with parton showers.
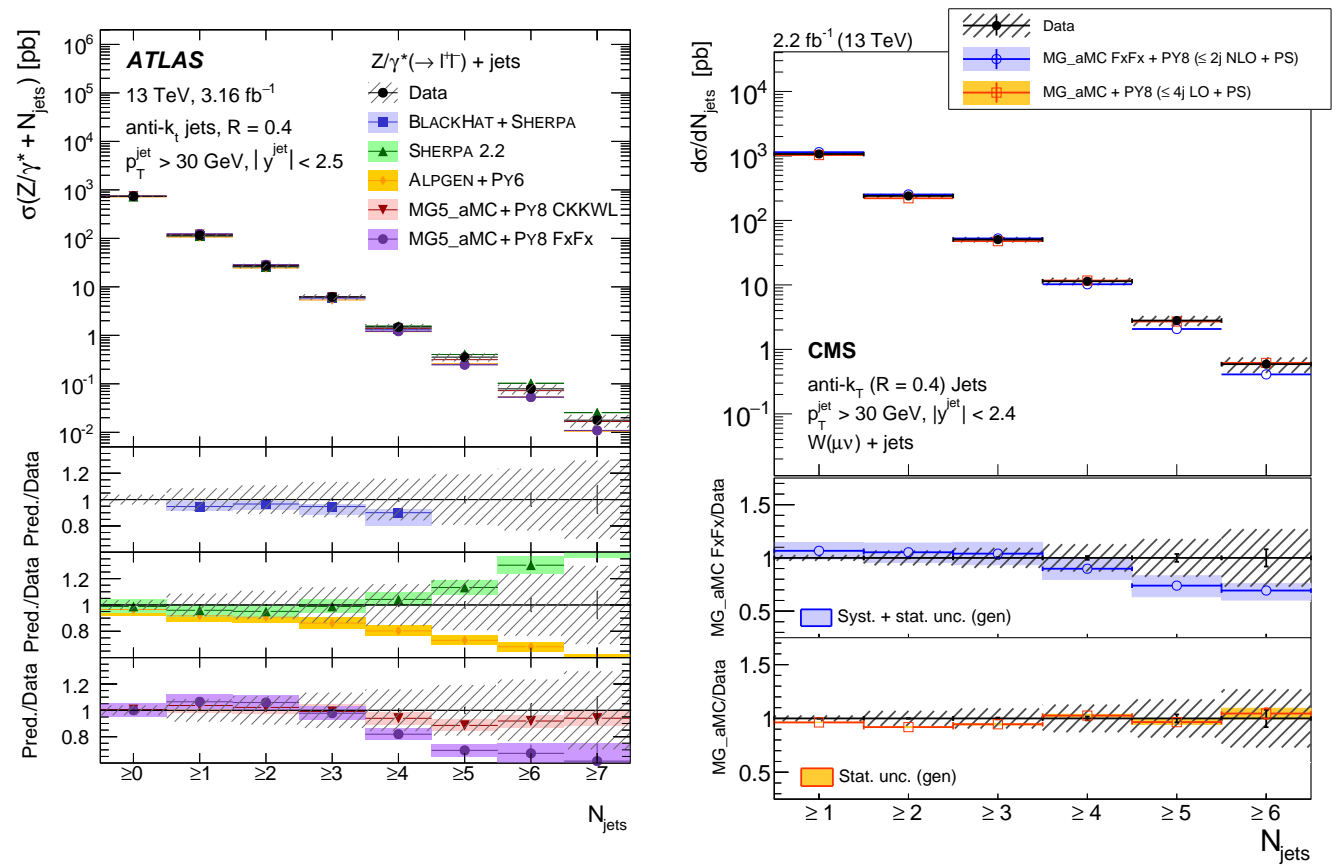

Figure 14: Left: Measured cross section as a function of the inclusive jet multiplicity for inclusive Z+jets events using ATLAS $13 \mathrm{TeV}$ data and comparison with various theoretical predictions [32]. Right: Differential $\mathrm{W}+$ jets cross section measurement for the exclusive jet multiplicity using CMS $13 \mathrm{TeV}$ data and comparison to predictions [33].

Of course, more information can be obtained from differential cross section measurements in which a specific jet multiplicity is selected. For example, Figure 15 presents the results of some 
of the $\mathrm{W}+$ jets differential cross section measurements made with ATLAS $8 \mathrm{TeV}$ data [34]. From these results we see that while a 2-to-2 leading order matrix-element calculation interfaced to a parton shower is not sufficient to account for hard parton radiation, fixed-order matrix-element calculations are also not sufficient to adequately predict the distribution of many observables. For example, the $H_{T}$ variable, presented on the left panel of Figure 15, is badly modeled by the NLO Black Hat [35] calculation, and even by the NNLO $\mathrm{N}_{\text {jetti }}$ calculation [36]. It is therefore important to combine both a hard QCD calculation from higher order matrix elements with a soft QCD modeling from a parton shower to properly describe observables like $H_{T}$. However, this approach includes a new source of variations in the predictions: the matching scheme. As can be seen in the right panel of Figure 15, there is a large discrepancy between LO and NLO Sherpa predictions after merging/matching the NLO matrix elements to the Sherpa parton shower, with data surprisingly favoring the LO calculations. Could it be due to a problem with the NLO matching procedure? It is certainly possible, but more investigations are needed to support this conclusion.
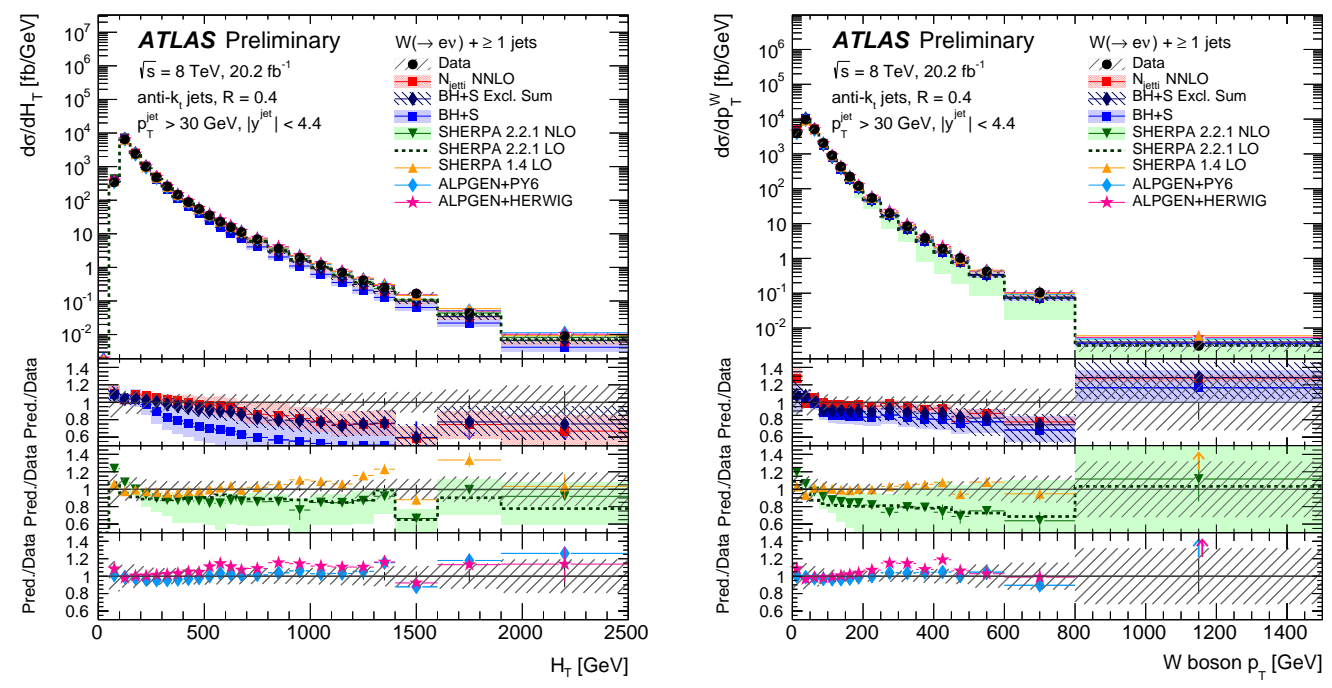

Figure 15: Differential cross sections measured by the ATLAS Collaboration on $8 \mathrm{TeV}$ data for the production of a W-boson as a function of $H_{T}$ (left) and $p_{T}^{W}$ (right) for events with at least one jet [34].

Further uncertainties affect theoretical predictions when, in addition to the exclusive jet selections, heavy flavor requirements are added. Measurements of the W- and Z-boson production accompanied by heavy flavor jets are sensitive to the heavy flavor content of the PDFs, which is not as precisely known as the light-quark content. Production of such jets through gluon-splitting in parton showers is another potential source of discrepancy. The mass of the heavy quarks constitutes a natural cut-off, which pushes the kinematics of the splitting partons away from the soft and collinear region of the phase space, where parton showers are valid. They also cannot account for the 2-to-3 processes, where two heavy flavor quarks are observed in the final state, and where the Z-boson is emitted from one of them. Finally, the effect of the heavy-flavour quark mass is not consistently accounted for in all matrix-element calculations. Some predictions directly take the production of heavy flavor jets from the heavy flavor quarks included in the PDFs. We say 
that these predictions have been obtained in the 5 flavor scheme (5FNS). The advantage of this approach is that the $\log \left(\frac{M_{Z}^{2}}{m_{b}^{2}}\right)$ terms are directly resummed in the DGLAP equation, and it simplifies the calculation, removing the need for $m_{b} \neq 0$ in the matrix elements. Another approach to heavy flavor jet production considers only 4 flavors in the PDFs (4FNS) and getting the massive b-quark flowing through one of the initial fermion lines of the process of interest coming from a gluon-splitting. A fully consistent treatment of this approach requires NLO calculations. Heavy-flavor jets produced from final state gluon-splitting are however modeled in the same way in both schemes, therefore mixing the sources of heavy-flavor jets. The $7 \mathrm{TeV}$ ATLAS W+b-jet measurements [37], presented in Figure 16, indicate a tension between data and NLO MCFM [38] predictions. This tension increases with the $p_{T}$ of the B-jet, and therefore seems to be genuine. However, it is a very challenging measurement, and the uncertainties are large, which hinders the conclusions.

The conclusions seem more straightforward for measurements of the $\mathrm{Z}+\mathrm{b}$-jet production [39, 40] because there is no flavor mixing in the neutral current at tree-level in the SM. As can be seen on the left panel of Figure 17, ATLAS results demonstrated that there is a good agreement between the data and the 5 flavor scheme predictions at NLO, but an important discrepancy with the NLO 4 flavor scheme prediction as well as with all of the LO predictions. However, CMS results, presented on the right panel of Figure 17, indicate that NLO matrix element calculations, matched to a parton shower in the 5 flavor scheme, might not be as good as one would expect. These predictions significantly deviate from the data for small b-jet momenta where the effect of the b-quark mass is relatively important. It is also interesting to observe that if two heavy flavor jets are required in the final state of $Z+j$ jets events, as we can see on the left panel of Figure 18, it is the 4 FNS prediction that is found to be in a better agreement with the measurement results. Moreover, the choice of flavor scheme used for the predictions seems to be irrelevant to the modeling of the angular distance between the two b-jets. As can be seen on the left panel of Figure 18, all LO matrix-element plus parton-shower predictions do not describe well the data in kinematic regions where the two b-jets are expected to be close to each other. On the other hand, when the higher scale gluon-splitting is analytically modeled in an NLO matrix element calculation, the description of the $\Delta \phi_{b b}$ observable is much better. This is a clear indication of the limits of parton showers in accurately describing gluon splitting into heavy flavor quarks and of the full matrix-element calculations that are needed to account for this. More precise measurements are however still needed in order to fully understand the impact of heavy flavor quarks on PQCD predictions.

\section{Measurement of Processes Dominated by the Electroweak Interaction}

So far, the discussion was concentrated on QCD SM measurements at the LHC. It is now time to turn to the electroweak SM physics program of the ATLAS and CMS Collaborations. The first class of measurements to be considered are the W- and Z-boson production in which there is either no hard QCD vertex, or there is a multiple gauge-boson vertex. This is for example the case in vector boson fusion (VBF) processes. Lepton+jets production in VBF processes constitute an important source of background to many Higgs measurements or searches for new physics. These events are characterized by two forward jets with a large rapidity separation. Such processes (together with other electroweak vector boson production with forward jets) have been observed 

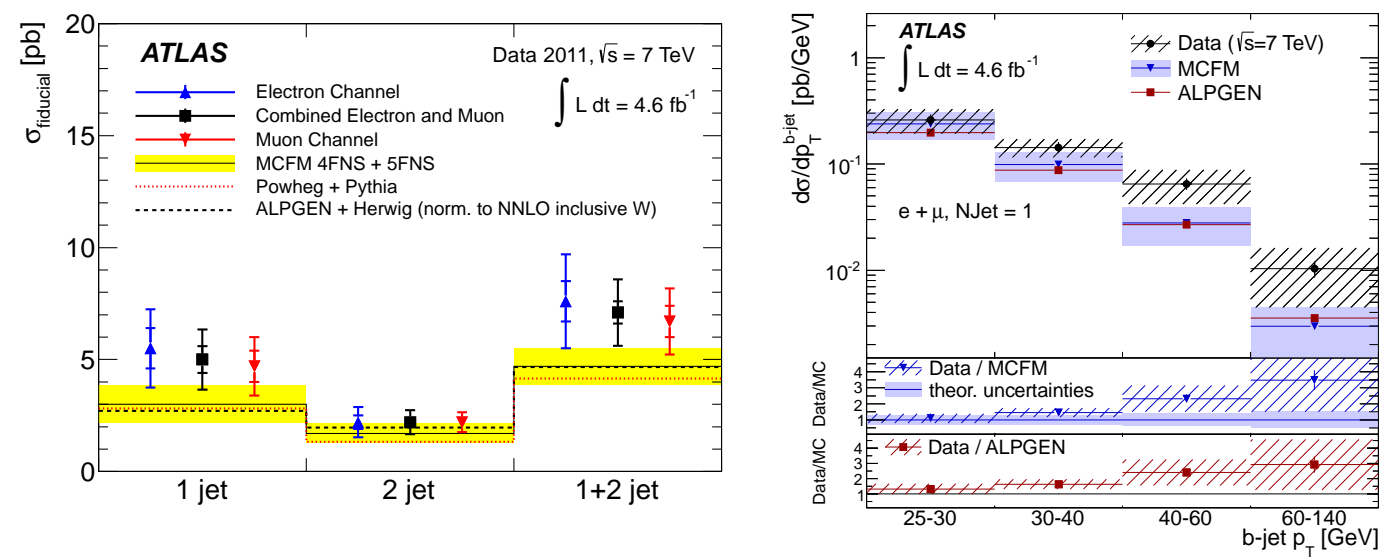

Figure 16: Left: Measured W+b-jet fiducial cross-sections in the electron, muon, and combined electron and muon channels for exactly one, two, and one or two jets (only one of which is a b-jet) in the event. The measurement results are compared with NLO predictions calculated with MCFM (corrected for hadronization and double-parton interaction effects), as well as with NLO prediction from Powheg interfaced to Pythia, and LO Apgen predictions interfaced to Herwig and Jimmy and scaled by a NNLO inclusive W normalization factor. Right: Measured differential W+b-jets cross-sections as a function of the $p_{T}$ of the b-jet when there is exactly one jet in the event, combining the muon and electron channel results. The measurement results are compared to the MCFM and to the Alpgen+Herwig+Jimmy predictions. Both results have been obtained with $7 \mathrm{TeV}$ ATLAS data [39].
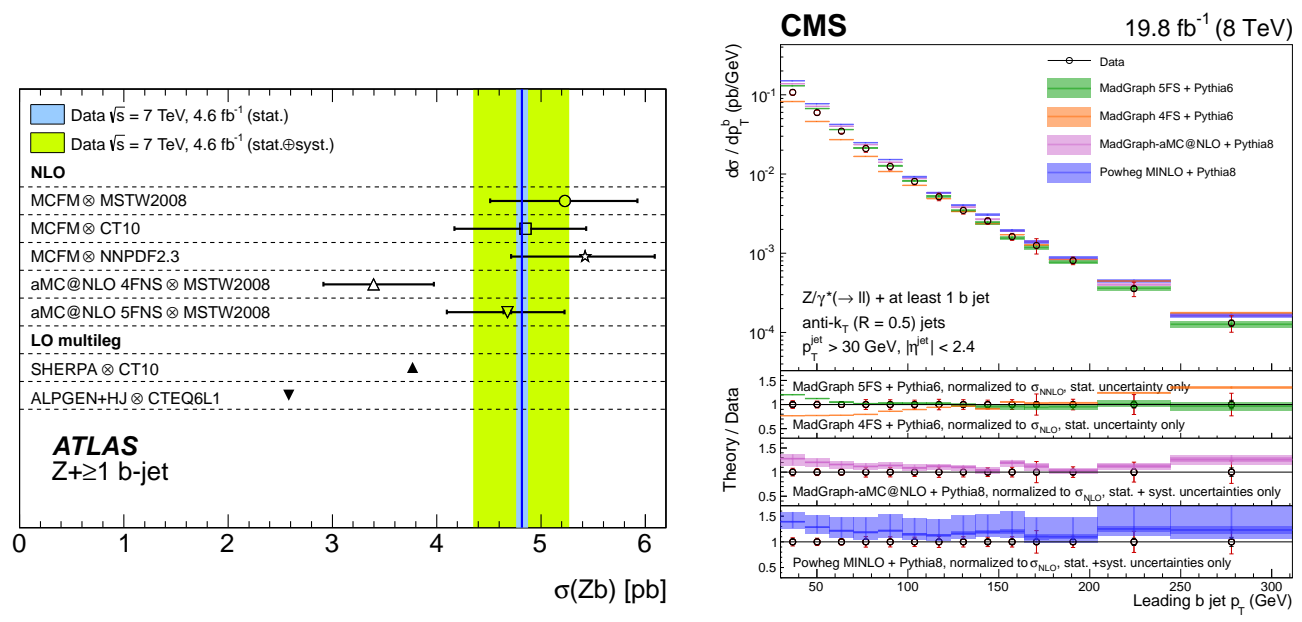

Figure 17: Left: Inclusive $b$-jet cross-sections for $Z+\geq 1$ b-jet events measured on 7 TeV ATLAS data [39]. Comparisons are made to NLO predictions from MCFM interfaced to different PDF sets and to aMC@NLO interfaced to the same PDF set in both the 4FNS and 5FNS. Comparisons are also made to LO multilegged predictions from Alpgen+Herwig+Jimmy and Sherpa. Right: Differential fiducial cross section for $\mathrm{Z}(1 \mathrm{~b})$ production as a function of the leading b-jet $p_{T}$ compared with the MadGraph 5FS, MadGraph 4FS, MadGraph5 aMC@NLO, and Powheg MinLO theoretical predictions. These results have been obtained with the CMS $8 \mathrm{TeV}$ data [40]. 

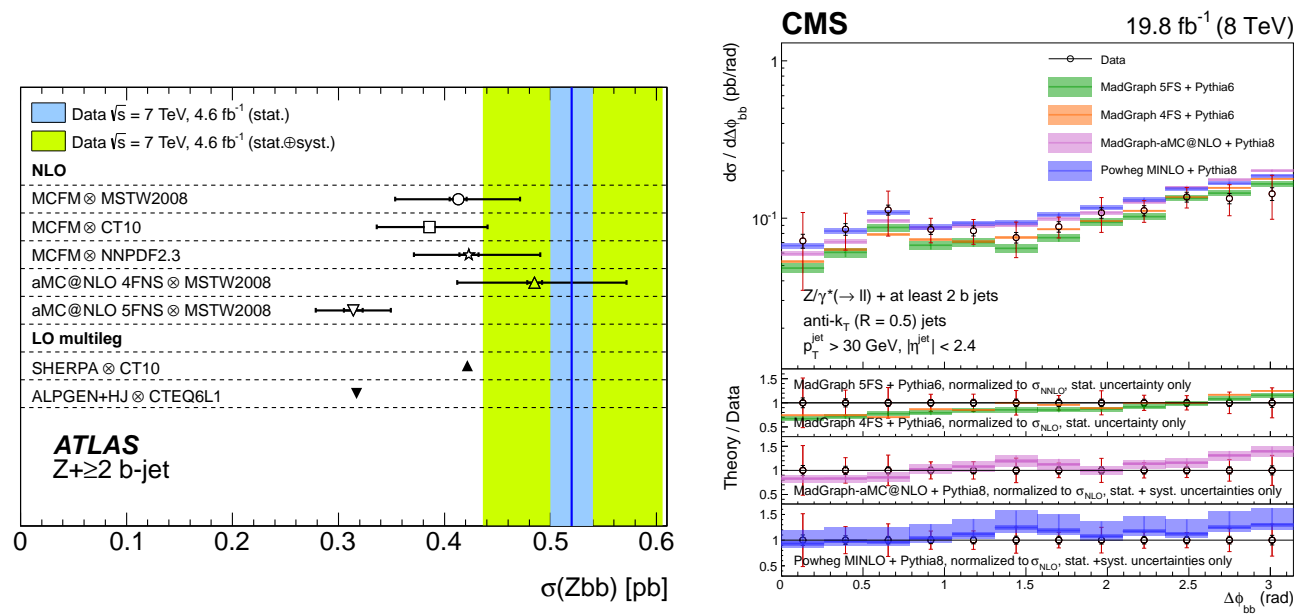

Figure 18: Left: Inclusive b-jet cross-sections for $\mathrm{Z}+\geq 2$ b-jet events measured on $7 \mathrm{TeV}$ ATLAS data [39]. Comparisons are made to NLO predictions from MCFM interfaced to different PDF sets and to aMC@NLO interfaced to the same PDF set in both the 4FNS and 5FNS. Comparisons are also made to LO multilegged predictions from Alpgen+Herwig+Jimmy and Sherpa. Right: Differential fiducial cross section for $\mathrm{Z}(1 \mathrm{~b})$ production as a function of $\Delta \phi_{Z b}$ compared with the MadGraph 5FS, MadGraph 4FS, MadGraph5 aMC@NLO, and Powheg MinLO theoretical predictions. These results have been obtained with the CMS 8 TeV data [40].

in many different final states, sometimes with a signal significance exceeding $5 \sigma$. As can be seen in Figure 19, both ATLAS and CMS data are globally in good agreement with predictions. These processes being relatively rare, their experimental uncertainties are significantly larger than the theory uncertainties. Testing the relevant SM physics requires further measurements.

The CMS and ATLAS Collaborations nevertheless succeeded in non-ambiguously observing the electroweak contribution to W+2-jets events, despite a large QCD contribution [41, 44]. This is illustrated in Figure 20. The left panel presents a comparison between CMS data and predictions from a purely electroweak contribution to the invariant mass of the two leading jets in $\mathrm{W}+2$-jets events. The good agreement between data and predictions therefore implies that the purely electroweak contribution to vector boson production in the VBF phase space is directly observable and is well described by the theory. Similarly, the right panel shows that the QCD-only predictions are insufficient at properly describing ATLAS W+2-jet data in the VBF phase space, and that the electroweak contribution is needed. It will soon be possible to study the interplay between electroweak and QCD higher order corrections. All this is important because the electroweak contribution to VBF processes increases relative to the QCD contribution when the vector boson momentum increases, i.e., in the phase space regions where new physics will soon be searched for.

Electroweak processes can also be carefully studied in events involving the production of multiple vector bosons. There is a plethora of diboson (VV') cross section measurements, which probe electroweak couplings with high precision: $\mathrm{V}$ and $\mathrm{V}^{\prime}$ can be a $\mathrm{W}, \mathrm{a} \mathrm{Z}$ or a photon, with the massive gauge boson decaying to electrons (ee or $e v$ ), muons $(\mu \mu$ or $\mu v)$, or neutrinos $(v v)$. These cross sections have been measured at $7 \mathrm{TeV}, 8 \mathrm{TeV}, 13 \mathrm{TeV}$ center-of-mass energy, leading to over 60 different possible diboson measurements in each of the ATLAS and CMS collaborations. 


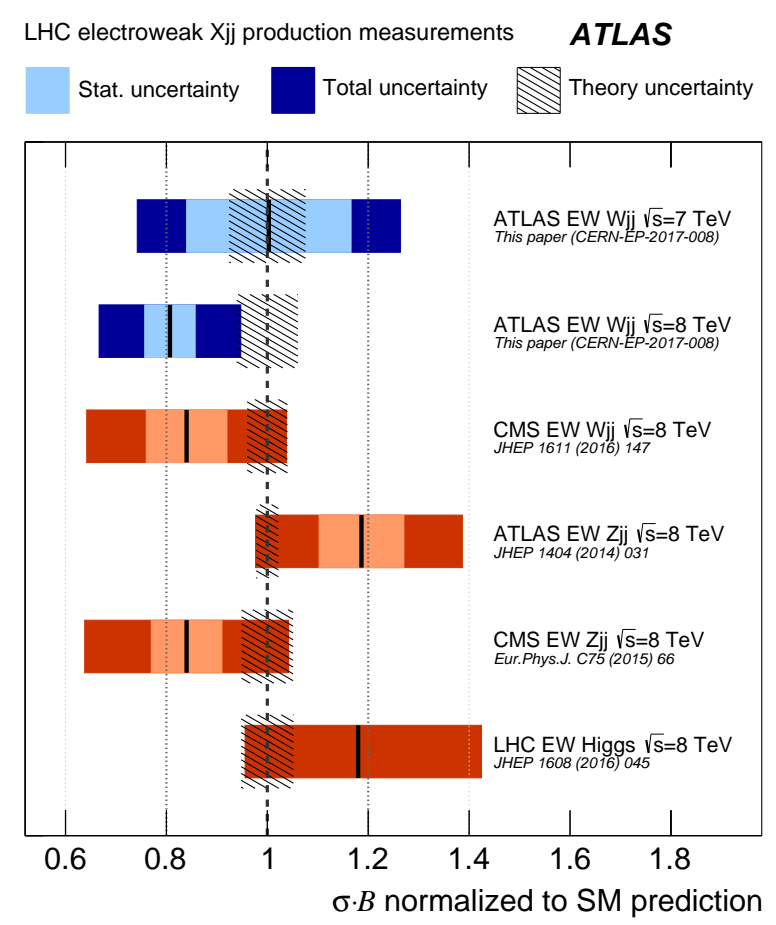

Figure 19: Measurements of the cross section times branching fractions of electroweak production of a single W, Z, or Higgs boson at high dijet invariant mass in a VFB event topology, divided by the SM predictions (Powheg+Pythia8 for ATLAS, Madgraph+Pythia8 for CMS, and Powheg+Pythia8 for the LHC combination) [41].

These different cross sections span many orders of magnitude, as can be seen in Figure 21. The latest measurement results are challenging NLO predictions, and most results are found in excellent agreement with NNLO calculations, as demonstrated by both collaborations with the two plots of Figure 22. So far, only two of the many obtained results feature some tension with the predictions. The experimental communities have now started exploring diboson differential cross sections.

As discussed in the introduction, the electroweak processes involve some Feynman diagrams with a triple gauge coupling, which can therefore be used to constrain generic new physics with effective Lagrangians. There are multiple coupling parameters to be constrained, depending on the higher-order effective operators considered. So far, no evidence for a significant deviation with respect to the SM has been observed, and tight constraints have been put on various effective operators. Figure 23 summarizes the combined ATLAS and CMS limits on the anomalous triple gauge coupling (aTGC) from ZZ SM measurements [45].

One of the biggest SM electroweak experimental challenges is however the measurement of vector boson scattering (VBS). It consists of a VBF process with a diboson final state. It provides a unitarity test of the electroweak sector, because longitudinally polarized diboson VBS amplitudes require a Higgs boson in order to satisfy unitarity. Currently, no observation of a significant SM signal has yet been made, but the experimental collaborations are close to an observation. These measurements are however already valuable because BSM models enhance the VBS signal with an 

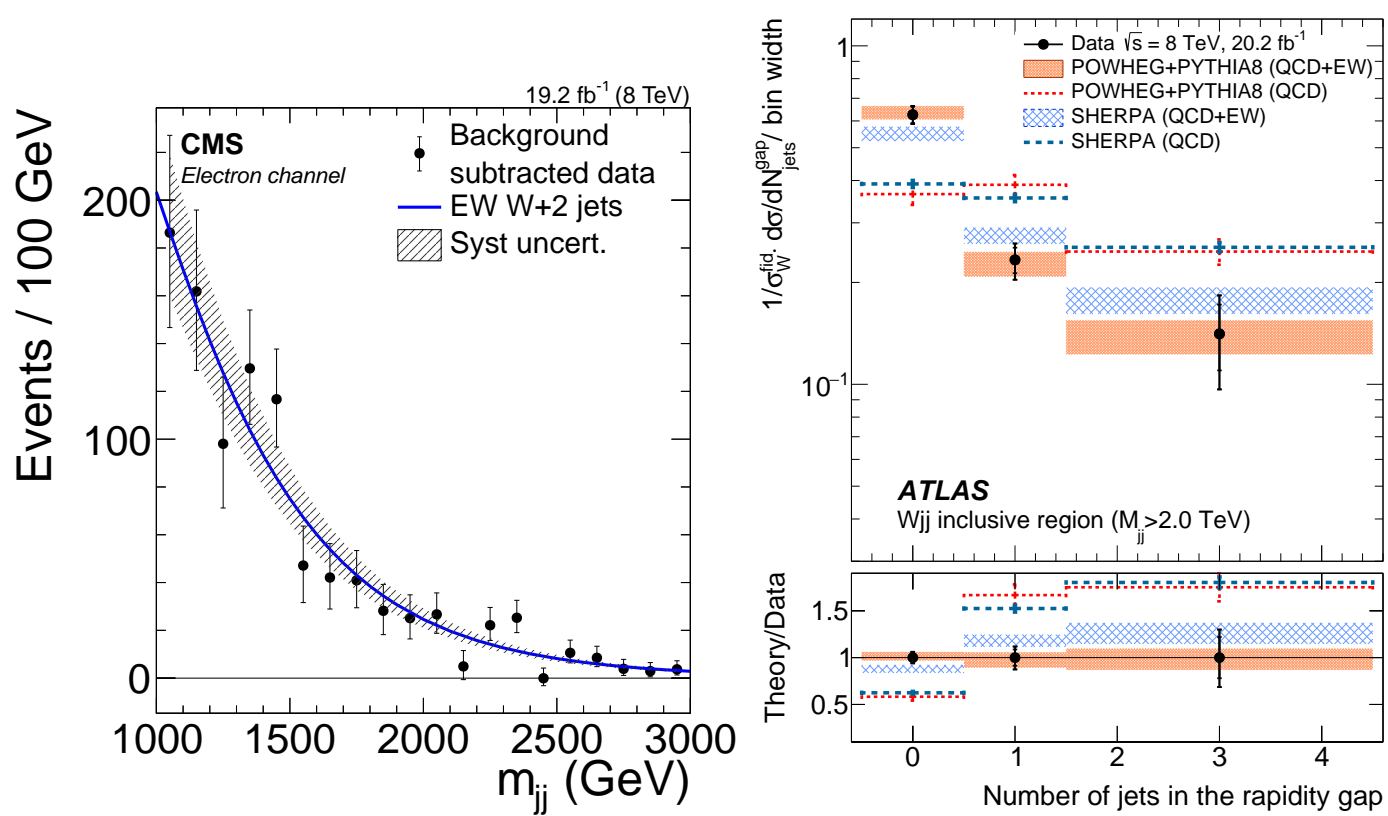

Figure 20: Left: Distribution of the two-jet invariant mass $M_{j j}$ for $\mathrm{Wjj}$ events in the electron channel obtained from $8 \mathrm{TeV}$ CMS data [44]. The $M_{j j}$ distribution is shown after subtracting all components except the EW W+2-jets process. The predictions are obtained with MadGraph5-aMC@NLO 2.1 interfaced to PYTHIA 6.4. Right: Unfolded normalized distribution of the number of jets with $p_{T}>30 \mathrm{GeV}$ in the rapidity interval bounded by the two highest- $p_{T}$ jets in the inclusive fiducial region of $\mathrm{Wjj}$ events with a $\mathrm{M} M_{j j}$ threshold of $2 \mathrm{TeV}$. The results have been obtained with ATLAS 7 and $8 \mathrm{TeV}$ data [41]. Comparisons are made to QCD Wjj and QCD+EWK Wjj NLO predictions obtained from Powheg+Pythia and Sherpa 2.2.

anomalous quartic gauge coupling (aQGC) at high transverse momentum of the vector bosons and at high invariant mass of the diboson system. A few different BSM constraints have been obtained from such VBS measurements [46], the best ones being summarized in Figure 24.

\section{Measurements of Electroweak Parameters}

Finally, precise measurements of the main electroweak parameters of the SM have high scientific value, as discussed in the introduction. Recent experimental efforts have been dedicated to the measurements of the weak mixing angle $\left(\sin ^{2} \theta_{W}\right)$ and of the mass of the W-boson. The weak mixing angle is measured using a forward-backward asymmetry in $\mathrm{Z}$ events. Forward and backward events are defined in the Collins-Soper frame by: $\cos \theta^{\star}=\frac{2\left(p_{1}^{+} p_{2}^{-}-p_{1}^{-} p_{2}^{+}\right)}{\sqrt{M^{2}\left(M^{2}+P_{T}^{2}\right)}} \times \frac{P_{Z}}{\left|P_{Z}\right|}$. The weak mixing angle is extracted from the asymmetry measurement using invariant mass $\left(m_{l l}\right)$ templates, where the vector couplings of leptons to the $\mathrm{Z}$ boson are varied. As can be seen on the left panel of Figure 25, the measured forward-backward asymmetry obtained by the CMS Collaboration with 8 $\mathrm{TeV}$ data agrees well with predictions [47]. A summary of various results on the measurement of $\sin ^{2} \theta_{W}$ is presented on the right panel of Figure 25. The precision reached at the LHC [48] is still not competitive with the LEP results, but with more data, and with an ATLAS, CMS, and LHCb combination, which would offset many experimental systematic uncertainties, it is not impossible 


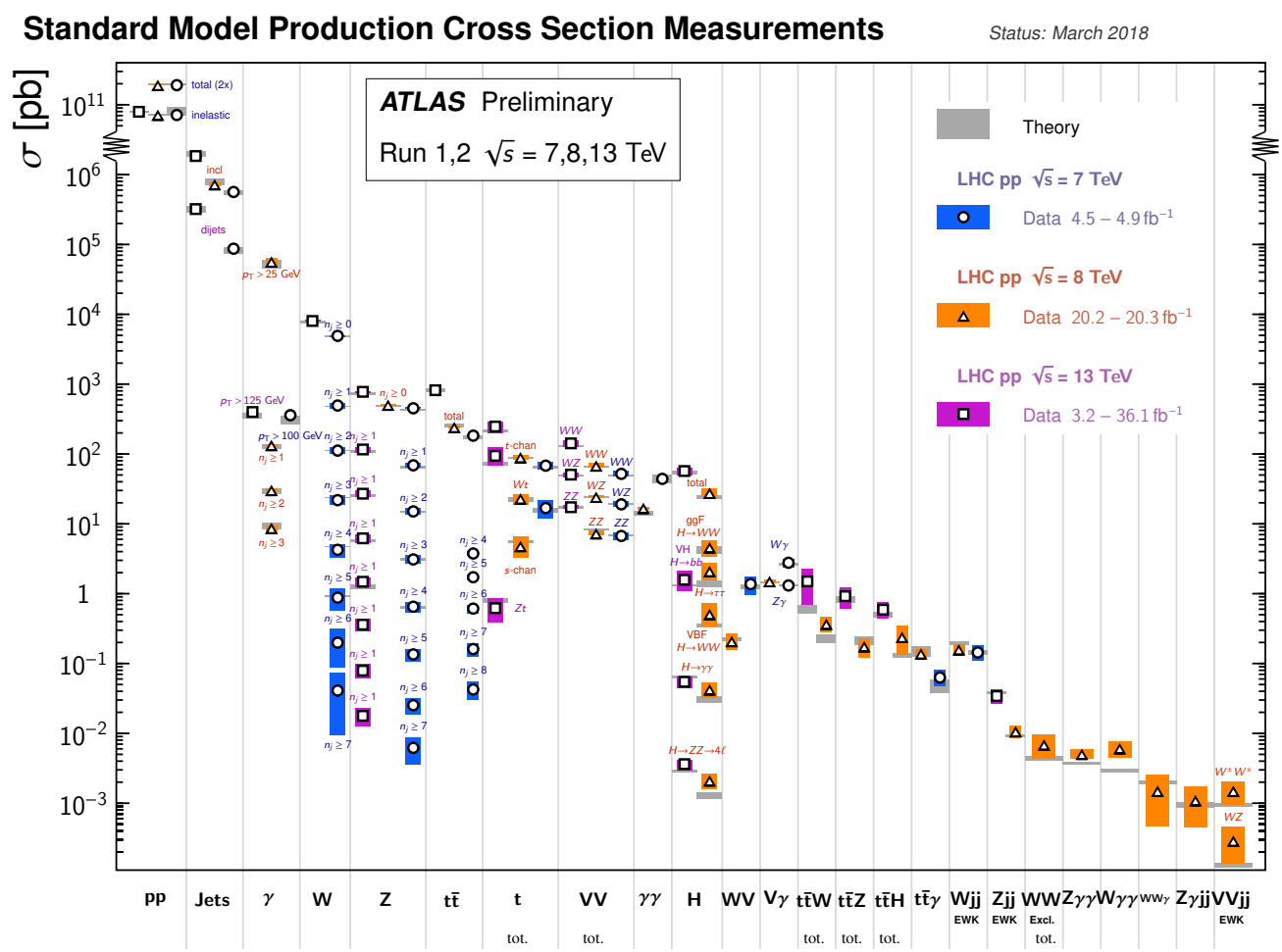

Figure 21: Summary of several Standard Model total and fiducial production cross section ATLAS measurements [42], corrected for leptonic branching fractions, compared to the corresponding theoretical expectations. All theoretical expectations were calculated at NLO or higher. The luminosity used for each measurement is indicated close to the data point. Some measurements have been extrapolated using branching ratios as predicted by the Standard Model for the Higgs boson.
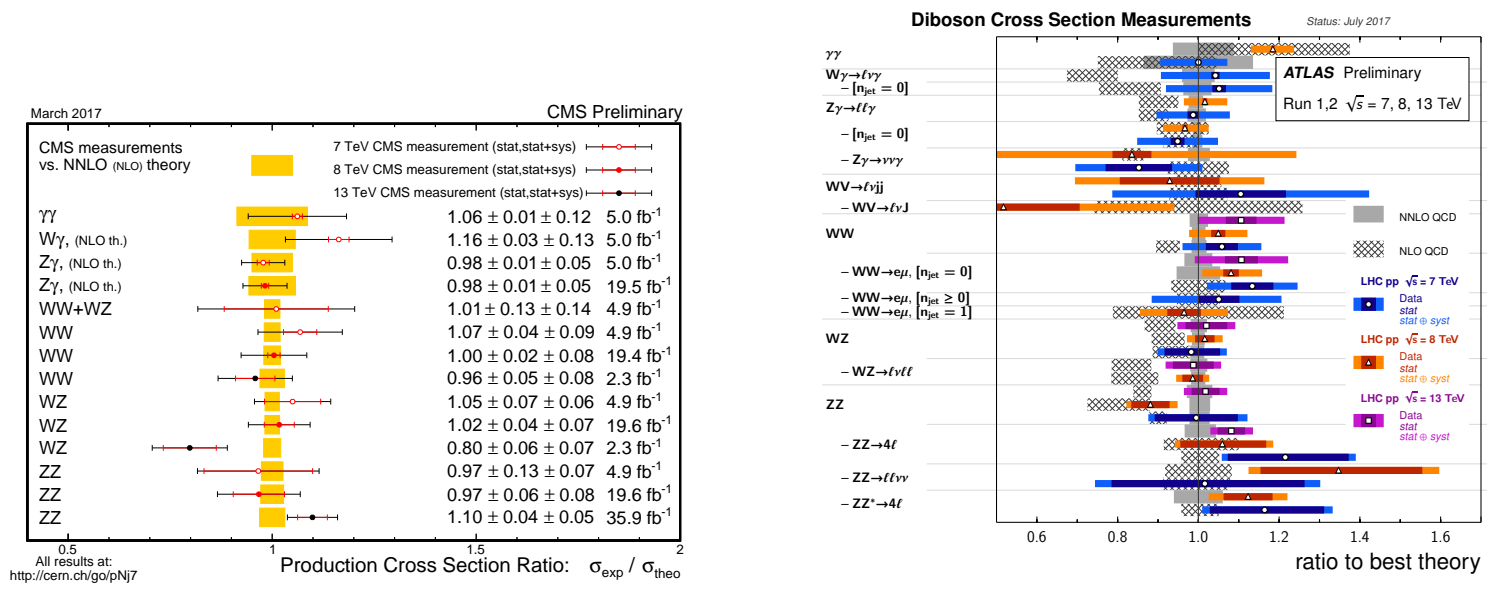

Figure 22: Left: Diboson cross section ratio comparison to theory: theory predictions updated to latest NNLO calculations where available compared to predictions in the CMS papers and preliminary physics analysis summaries [43]. Right: The ratio for several diboson total and fiducial production cross section ATLAS measurements over the best available theory prediction, corrected for leptonic branching fractions [42]. 


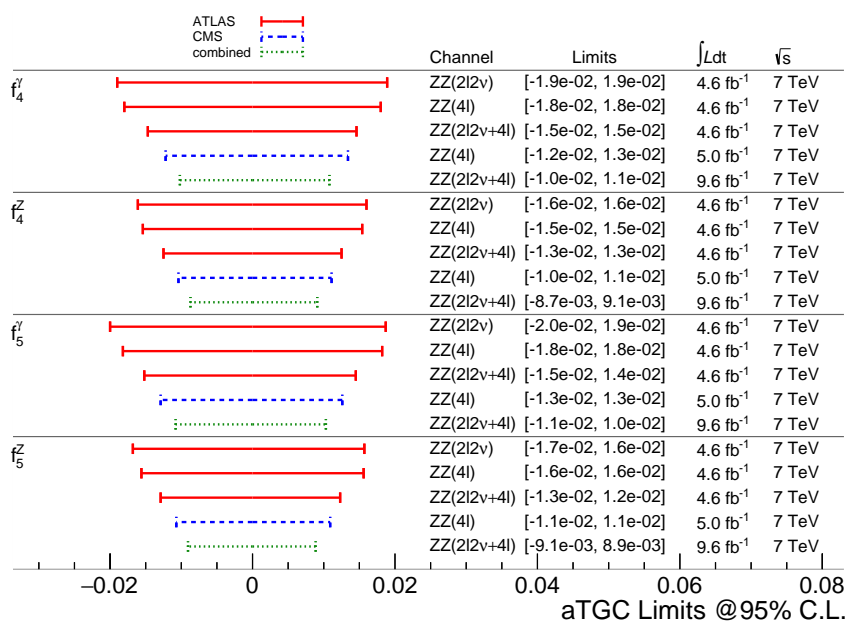

Figure 23: Combination of ATLAS and CMS experiment results on ZZ production measurements to obtain constraints on anomalous neutral triple gauge boson couplings. The limits are on aTGCs one-parameter for an effective theory cut-off scale set to $\Lambda=\infty$. All anomalous coupling parameters other than the measured one are set to their SM values. The datasets correspond to integrated luminosities of 4.6 and $5.0 \mathrm{fb}^{-1} \mathrm{for}$ ATLAS and CMS, respectively. The combination is performed in the fully leptonic decay channels. Details of the combination procedures are discussed in [45].

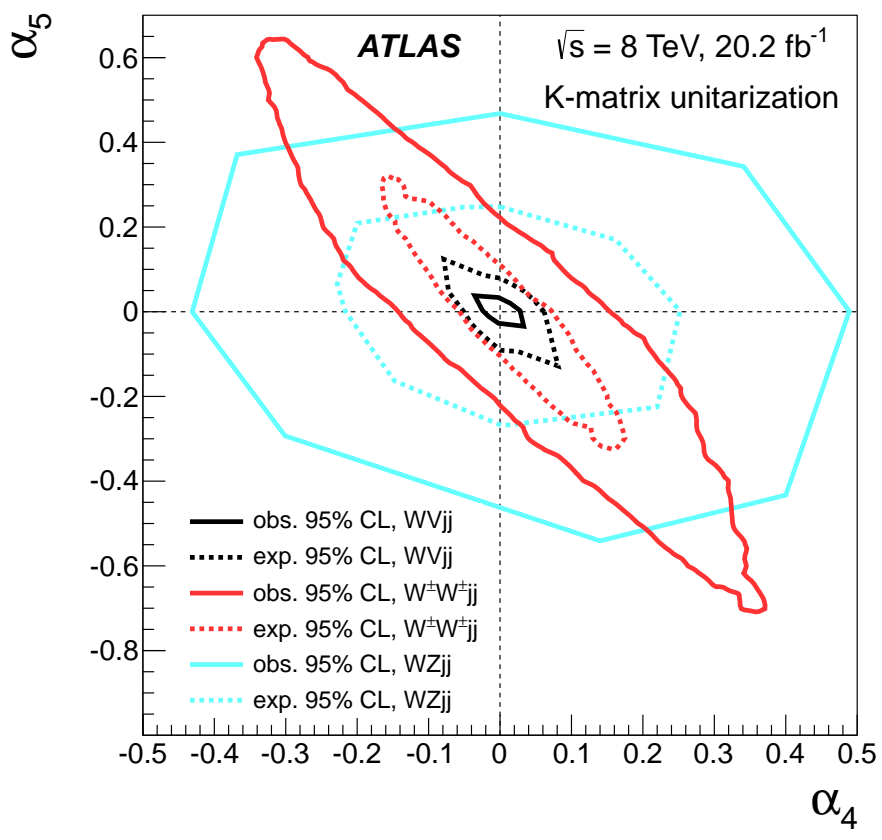

Figure 24: The observed 2D confidence region (solid black contour) for two anomalous quartic gauge boson coupling parameters $\alpha_{4}$ and $\alpha_{5}$, at 95\% CL. Results from different ATLAS VBS production analyses are compared: WW/WZ semi-leptonic channel (in black), $\mathrm{W}^{ \pm} \mathrm{W}^{ \pm}$(in red) and WZ (in cyan) [46]. 
that the LHC will eventually reach the level of precision attained at LEP. That would allow for stringent consistency tests of the SM.
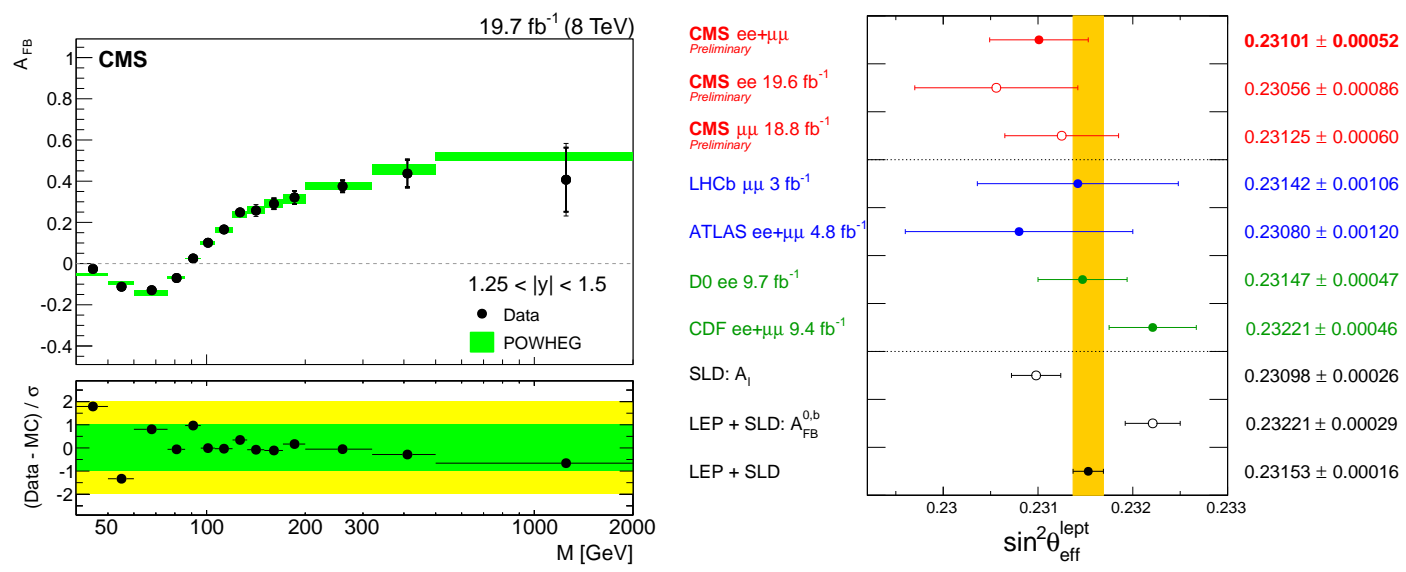

Figure 25: Left: The combined $\left(\mu^{+} \mu^{-}\right.$and $\left.e^{+} e^{-}\right)$unfolded forward-backward asymmetry (AFB) distributions for Z rapidity $1.25<|y|<1.5$ obtained from $8 \mathrm{TeV}$ CMS data [47]. The measurement result is compared with a prediction obtained with the Powheg generator. Right: Comparison of the measured $\sin ^{2} \theta_{\text {eff }}^{\text {lept }}$ in the muon and in the electron channels and their combination with the previous LEP, SLC, Tevatron and LHC measurements [48]. The shaded band corresponds to the combination of the LEP and SLC measurements.

The ATLAS experiment recently published the first LHC W mass measurement [49]. The level of precision reached with this measurement is comparable with the precision obtained by the CDF collaboration a few years ago. This can be seen on the right panel of Figure 26. This measurement is very challenging because it requires several precise and sophisticated ancillary measurements to pin down the various systematic uncertainties affecting the results. Most of the measurements presented above have, in one way or another, contributed to the precision of the W-mass measurement. ATLAS reached a stunning $0.024 \%$ precision on $M_{W}$. Such a result can be used in consistency tests, as discussed in the introduction. Current result indicates an excellent agreement between W, top and Higgs mass measurements, as can be seen on the right panel of Figure 26.

\section{Conclusion}

QCD and electroweak phenomena are pervasive elements of particle physics. They are the subject of a variety of challenging experimental measurements, a small subset of which has been shown in this proceeding. Each measurement usually delivers an important message to the experimental and theoretical communities. Comparison of the results against the state-of-the-art theory predictions have indicated that theoretical improvements are needed to address some of the observed tensions. On the other hand, the measurements have been used to reach improved understandings of the proton structure, of soft and hard QCD radiation, of electroweak contribution to various vector boson production modes, and of the gauge structure of the SM, including the possibility of anomalous triple and quartic gauge couplings. These measurements help reduce uncertainties, therefore improving the general sensitivity of LHC experiments to new physics. The 

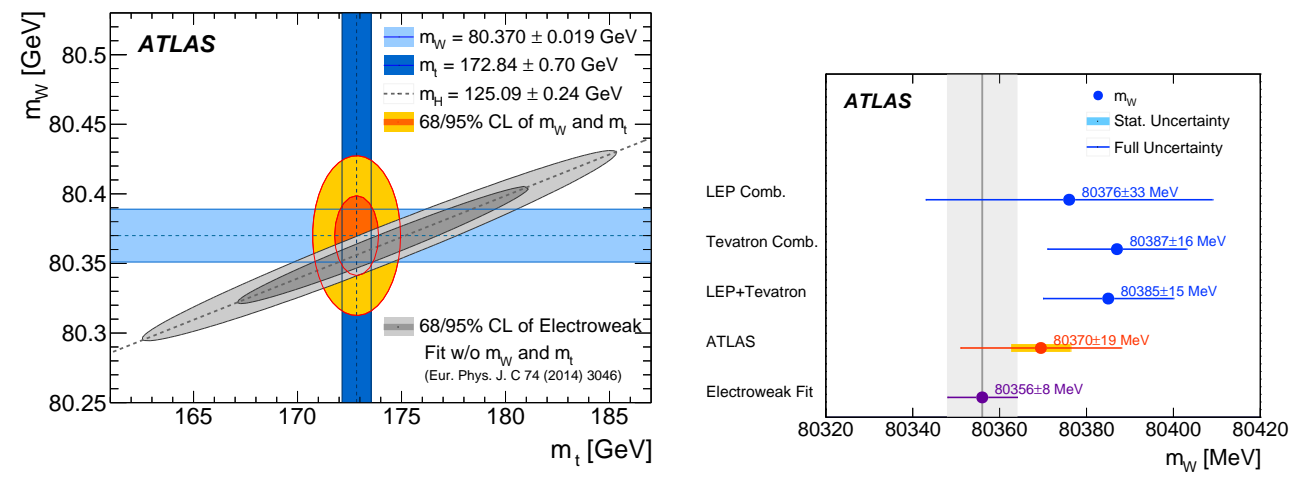

Figure 26: Left: The ATLAS measured value of $M_{W}$ [49] compared with other published results, including measurements from the LEP and the Tevatron collider experiments. The determination from the electroweak fit uses as input the LHC measurement of the Higgs-boson mass, $m_{H}=125.09 \pm 0.24 \mathrm{GeV}$ [50]. Right: The $68 \%$ and $95 \%$ confidence-level contours of the $M_{W}$ and $m_{t}$ indirect determination from the global electroweak fit are compared to the $68 \%$ and $95 \%$ confidence-level contours of the ATLAS measurements of the top-quark and W-boson masses.

LHC SM physics program is far from being exhausted, new measurements are still needed. Mastering QCD and electroweak phenomena is both essential for the future of the LHC program and for the advancement of our knowledge.

\section{References}

[1] The ATLAS Collaboration, Observation of a new particle in the search for the Standard Model Higgs boson with the ATLAS detector at the LHC, Phys. Lett. B 716 (2012) 1-29.

[2] The CMS Collaboration, Observation of a new boson at a mass of $125 \mathrm{GeV}$ with the CMS experiment at the LHC, Phys. Lett. B 716 (2012) 30.

[3] D. Hanneke, S. Fogwell, G. Gabrielse, New measurement of the electron magnetic moment and the fine structure constant, Phys. Rev. Lett. 100 (2008) 120801; G. Gabrielse, The standard models greatest triumph, Physics Today 66, 12 (2013) 64.

[4] C. Patrignani et al. (Particle Data Group), The Review of Particle Physics, Chin. Phys. C 40 (2016 and 2017 update) 100001.

[5] J. M. Campbell, J. Huston, and W. Stirling, Rept. Prog. Phys. 70 (2007) 89.

[6] R. Ellis, W. Stirling, and B. Webber, QCD and Collider Physics, Cambridge University Press (1996).

[7] A. Buckley et al., General-purpose event generators for LHC physics, arXiv:1101.2599 [hep-ph].

[8] M. E. Peskin, and T. Takeuchi, Estimation of oblique electroweak corrections, Phys. Rev. D 46 (1992) 381 .

[9] T. Sjöstrand, S. Mrenna and P. Z. Skands, A Brief Introduction to PYTHIA 8.1, Comput. Phys. Commun. 178 (2008) 852?867; T. Sjöstrand, S. Mrenna and P. Z. Skands, PYTHIA 6.4 Physics and Manual, JHEP 0605 (2006) 026. 
[10] M. Bähr et al., Herwig++ Physics and Manual, Eur. Phys. J. C 58 (2008) 639?70; G. Corcella et al., HERWIG 6: An Event generator for hadron emission reactions with interfering gluons (including supersymmetric processes), JHEP 0101 (2001) 010.

[11] J. Alwall et al., The automated computation of tree-level and next-to-leading order differential cross sections, and their matching to parton shower simulations, JHEP 07 (2014) 079; R. Frederix and S. Frixione, Merging meets matching in MC@NLO, JHEP 12 (2012) 061; J. Alwall et al., MadGraph 5: going beyond, JHEP 06 (2011) 128.

[12] The CMS Collaboration, Measurements of inclusive 2-jet, 3-jet and 4-jet azimuthal correlations in pp collisions at $\sqrt{S}=13 \mathrm{TeV}$, CMS-PAS-SMP-16-014.

[13] The ATLAS Collaboration, Precision measurement and interpretation of inclusive $W+, W$ - and $Z / \gamma^{\star}$ production cross sections with the ATLAS detector, Eur. Phys. J. C 77 (2017) 367.

[14] The ATLAS Collaboration, Measurement of $W^{ \pm}$and Z-boson production cross sections in pp collisions at $\sqrt{S}=13 \mathrm{TeV}$ with the ATLAS detector, Phys. Lett. B 759 (2016) 601.

[15] The ATLAS Collaboration, Determination of the strange quark density of the proton from ATLAS measurements of the $W->l$ nu and Z -> ll cross sections, Phys. Rev. Lett. 109 (2012) 012001.

[16] The CMS Collaboration, Measurement of the differential cross section and charge asymmetry for inclusive $p p \rightarrow W^{ \pm}+X$ production at $\sqrt{S}=8 \mathrm{TeV}$, Eur. Phys. J. C 76 (2016) 469.

[17] The CMS Collaboration, Measurement of the double-differential inclusive jet cross section in proton-proton collisions at $\sqrt{S}=13 \mathrm{TeV}$, Eur. Phys. J. C 76 (2016) 451.

[18] The ATLAS Collaboration, Measurement of inclusive jet and dijet cross-sections in proton-proton collisions at $\sqrt{S}=13 \mathrm{TeV}$ with the ATLAS detector, arXiv:1711.02692 [hep-ex].

[19] The CMS Collaboration, Measurement of the triple-differential dijet cross section in proton-proton collisions at $\sqrt{S}=8 \mathrm{TeV}$ and constraints on parton distribution functions, Eur. Phys. J. C 77 (2017) 746.

[20] The ATLAS Collaboration, Measurement of the transverse momentum and $\phi_{\eta}^{\star}$ distributions of Drell-Yan lepton pairs in proton-proton collisions at $\sqrt{S}=8 \mathrm{TeV}$ with the ATLAS detector, Eur. Phys. J. C 76 (2016) 291.

[21] C. Balazs, J.-W. Qiu and C. Yuan, Effects of QCD resummation on distributions of leptons from the decay of electroweak vector bosons, Phys. Lett. B 355 (1995) 548-554; M. Guzzi, P. M. Nadolsky and B. Wang, Nonperturbative contributions to a resummed leptonic angular distribution in inclusive neutral vector boson production, Phys. Rev. D 90 (2014) 014030; M. Guzzi and P. M. Nadolsky, Nonperturbative contributions to a resummed leptonic angular distribution in inclusive $\mathrm{Z} / \gamma^{\star}$ boson production, Int. J. Mod. Phys. Conf. Ser. 20 (2012) 274 ?281.

[22] The CMS Collaboration, Measurement of the transverse momentum spectra of weak vector bosons produced in proton-proton collisions at $\sqrt{S}=8 \mathrm{TeV}$, JHEP 02 (2017) 096.

[23] S. Catani et al., Vector boson production at hadron colliders: a fully exclusive QCD calculation at NNLO, Phys. Rev. Lett. 103 (2009) 082001.

[24] The ATLAS Collaboration, Measurement of the $Z / \gamma^{\star}$ boson transverse momentum distribution in pp collisions at $\sqrt{S}=7 \mathrm{TeV}$ with the ATLAS detector, JHEP 09 (2014)145.

[25] S. Alioli et al., NLO vector-boson production matched with shower in POWHEG, JHEP 0807 (2008) 060; S. Alioli et al., A general framework for implementing NLO calculations in shower Monte Carlo programs: the POWHEG BOX, JHEP 06 (2010) 043. 
[26] S. Catani, Y. L. Dokshitzer, M. H. Seymour, and B. R.Webber, Longitudinally-invariant $k_{T}$-clustering algorithms for hadron-hadron collisions, Nucl. Phys. B 406 (1993) 187; S. D. Ellis, and D. E. Soper, Successive Combination Jet Algorithm For Hadron Collisions, Phys. Rev. D 48 (1993) $3160 ? 3166$.

[27] The ATLAS Collaboration, Measurement of kt splitting scales in $W \rightarrow$ lnu events at sqrt( $(s)=7 \mathrm{TeV}$ with the ATLAS detector, Eur. Phys. J. C 735 (2013) 2432.

[28] S. Höche, et al., A critical appraisal of NLO+PS matching methods, JHEP 09 (2012) 049; T. Gleisberg, et al., Event generation with SHERPA 1.1, JHEP 02 (2009) 007.

[29] The CMS Collaboration, Measurement of the differential jet production cross section with respect to jet mass and transverse momentum in dijet events from pp collisions at $\sqrt{S}=13 \mathrm{TeV}$, CMS PAS SMP-16-010.

[30] C. Frye, A. J. Larkoski, M. D. Schwartz, and K. Yan, Factorization for groomed jet substructure beyond the next-to-leading logarithm, JHEP 07 (2016) 064.

[31] S. Marzani, L. Schunk, and G. Soyez, A study of jet mass distributions with grooming, JHEP 07 (2017) 132.

[32] The ATLAS Collaboration, Measurements of the production cross section of a $Z$ boson in association with jets in pp collisions at $\sqrt{S}=13$ with the ATLAS detector, Eur. Phys. J. C 77 (2017) 361.

[33] The CMS Collaboration, Measurement of the differential cross sections for the associated production of a W boson and jets in proton-proton collisions at $\sqrt{S}=13 \mathrm{TeV}$, Phys. Rev. D 96 (2017) 072005.

[34] The ATLAS Collaboration, Measurement of differential cross sections and $W+/ W$ - cross section ratios for $W$ boson production in association with jets at sqrt(s) $=8 \mathrm{TeV}$ with the ATLAS detector, arXiv:1711.03296 [hep-ex].

[35] C. Berger et al., Next-to-leading order QCD predictions for W+3-jet distributions at hadron colliders, Phys. Rev. D 80 (2009) 074036; C. Berger et al., Precise predictions for $W+4$ jet production at the Large Hadron Collider, Phys. Rev. Lett. 106 (2011) 092001; Z. Bern et al., Next-to-leading order $W+5$ jet production at the LHC, Phys. Rev. D 88 (2013) 014025.

[36] R. Boughezal, C. Focke, X. Liu and F. Petriello, W-boson production in association with a jet at next-to-next-to-leading order in perturbative QCD, Phys. Rev. Lett. 115 (2015) 062002; A. Gehrmann-De Ridder, T. Gehrmann, E. W. N. Glover, A. Huss and T. A. Morgan, Precise QCD predictions for the production of a $Z$ boson in association with a hadronic jet, Phys. Rev. Lett. 117 (2016) 022001.

[37] The ATLAS Collaboration, Measurement of the cross-section for $W$ boson production in association with b-jets in pp collisions at sqrt $(s)=7 \mathrm{TeV}$ with the ATLAS detector, JHEP 06 (2013) 084.

[38] . M. Campbell and R. K. Ellis, An update on vector boson pair production at hadron colliders, Phys. Rev. D 60 (1999) 113006; J. M. Campbell, R. K. Ellis and D. L. Rainwater, Next-to-leading order $Q C D$ predictions for $W+2$ jet and $Z+2$ jet production at the CERN LHC, Phys. Rev. D 68 (2003) 094021 .

[39] The ATLAS Collaboration, Measurement of differential production cross-sections for a Z boson in association with b-jets in 7 TeV proton-proton collisions with the ATLAS detector, JHEP 10 (2014) 141.

[40] The CMS Collaboration, Measurements of the associated production of a $Z$ boson and bets in pp collisions at sqrt(s) = 8 TeV, Eur. Phys. J. C 77 (2017) 751. 
[41] The ATLAS Collaboration, Measurements of electroweak Wjj production and constraints on anomalous gauge couplings with the ATLAS detector, Eur. Phys. J. C 77 (2017) 474.

[42] The ATLAS Collaboration, Summary plots from the ATLAS Standard Model physics group, https://atlas.web.cern.ch/Atlas/GROUPS/PHYSICS/CombinedSummaryPlots/SM/

[43] The CMS Collaboration, Summary plots from the CMS Standard Model physics group, https://twiki.cern.ch/twiki/bin/view/CMSPublic/PhysicsResultsCombined

[44] The CMS Collaboration, Measurement of electroweak production of a $W$ boson and two forward jets in proton-proton collisions at $\sqrt{S}=8 \mathrm{TeV}$, JHEP 11 (2016) 147.

[45] The ATLAS and CMS Collaborations, Combination of results from the ATLAS and CMS experiments on anomalous triple gauge couplings in ZZ production from pp collisions at a centre-of-mass energy of $7 \mathrm{TeV}$ at the LHC, ATLAS-CONF-2016-036, CMS-PAS-SMP-15-001.

[46] The ATLAS Collaboration, Search for anomalous electroweak production of WW/WZ in association with a high-mass dijet system in pp collisions at $\sqrt{S}=8 \mathrm{TeV}$ with the ATLAS detector, Phys. Rev. D 95 (2017) 032001; The ATLAS Collaboration, Measurement of $W^{ \pm} W^{ \pm}$vector-boson scattering and limits on anomalous quartic gauge couplings with the ATLAS detector, Phys. Rev. D 96, (2017) 012007; The ATLAS Collaboration, Measurements of $W^{ \pm} Z$ production cross sections in p p collisions at $\sqrt{S}=8 \mathrm{TeV}$ with the ATLAS detector and limits on anomalous gauge boson self-couplings, Phys. Rev. D 93 (2016) 092004.

[47] The CMS Collaboration, Forward-backward asymmetry of Drell-Yan lepton pairs in pp collisions at $\sqrt{S}=8 \mathrm{TeV}$, Eur. Phys. J. C 76 (2016) 325.

[48] The CMS Collaboration, Measurement of the weak mixing angle with the forward-backward asymmetry of Drell-Yan events at $8 \mathrm{TeV}$, CMS-PAS-SMP-16-007.

[49] The ATLAS Collaboration, Measurement of the W-boson mass in pp collisions at $\sqrt{S}=7$ TeV with the ATLAS detector, Eur. Phys. J. C 78 (2018) 110.

[50] The ATLAS and CMS Collaborations, Combined Measurement of the Higgs Boson Mass in pp Collisions at $\sqrt{S}=7$ and 8 TeV with the ATLAS and CMS Experiments, Phys. Rev. Lett. 114 (2015) 191803. 